\title{
The development of a wax layer on the interior wall of a circular pipe transporting heated oil: the effects of temperature-dependent wax conductivity
}

\author{
Sophie Lauren Mason • John Christopher Meyer • David John Needham
}

Received: 21 April 2021 / Accepted: 8 September 2021 / Published online: 21 October 2021

(C) The Author(s) 2021

\begin{abstract}
In this paper, we develop and significantly extend the thermal phase change model, introduced in Needham et al. (QJMAM 67:93-125, 2014), describing the process of paraffinic wax layer formation on the interior wall of a circular pipe transporting heated oil, when subject to external cooling. In particular, we allow for the natural dependence of the solidifying paraffinic wax conductivity on local temperature. We are able to develop a complete theory, and provide efficient numerical computations, for this extended model. Comparison with recent experimental observations is made, and this, together with recent reviews of the physical mechanisms associated with wax layer formation provide significant support for the thermal model considered here.
\end{abstract}

Keywords Asymptotic limit · Generalised Stefan problem · Heated oil pipeline · Quasi-linear parabolic PDE . Wax layers

Mathematics Subject Classification $76 \mathrm{~T} 99 \cdot 80 \mathrm{~A} 22 \cdot 80 \mathrm{M} 35 \cdot 80 \mathrm{M} 20$

\section{Introduction}

In oil field operation, it is generally required that oil is transported in long sea bed pipelines. In a recent paper [1], one of the main undesirable features for pipeline oil transportation, namely the formation of paraffinic wax deposits on the inside of the pipe wall, was considered. This wax deposit happens when the temperature of the pipe wall falls below the wax solidification temperature, generally in the range $35-40^{\circ} \mathrm{C}$, but this can be in a wider range of $20-60{ }^{\circ} \mathrm{C}$, when dealing with real crude oils, rather than experimentally controlled oils. Notably, the accumulation of a wax deposit on a pipe wall effectively reduces the inner diameter of the pipe, which lessens the transport capacity of the pipe for a given driving pressure. A more detailed discussion of this phenomena is given in [1]. In particular, [1] was concerned with the introduction of a thermal phase change model (first proposed by Schulkes

S. L. Mason · J. C. Meyer $(\varangle) \cdot$ D. J. Needham.

School of Mathematics, University of Birmingham, Birmingham B15 2TT, UK

e-mail: J.C.Meyer@bham.ac.uk

S. L. Mason

e-mail: SLM585@student.bham.ac.uk

D. J. Needham

e-mail: D.J.Needham@bham.ac.uk 
in [2]) to capture the fundamental mechanism in the wax layer deposition process accurately. The fundamentals in this model are based on the assumption that the wax layer forms on cooling, as a thermal phase change from the dissolved wax in the hot oil, and therefore that the dynamics of the wax layer are dictated by thermal energy balances. The associated mathematical model was both formulated and analysed in detail in [1]. The outcomes were encouraging, in that a number of key observations in the wax layer deposition process, which were at odds with the historic material diffusion mechanism, were now fully accounted for in the thermal phase change model. This model has had further recent support in the independent works in [3] and [4]. ${ }^{1}$

In [1], a thermal model for the deposition of paraffinic wax on the inside of the pipe wall was proposed. The principal hypothesis introduced in [1] is that the key mechanism leading to wax deposition is a thermal phase change process. This led to a simple mathematical model based on balancing heat conduction from the heated oil, within the growing solid wax layer, and pipe wall, and into the coolant surrounding the exterior of the pipe. The mathematical formulation of this model gave rise to a free boundary problem (referred to as [IBVP] in [1]) of generalised Stefan type. This fundamental problem was analysed in considerable detail in [1]. A number of key salient features arising from the model were identified (see [1, Sect. 8, pp. 119-122]) with the intention of qualifying and quantifying the basis of the thermal model with detailed experiments performed by Hoffman \& Amundsen [5] and Halstensen et al. [6] amongst others. The experiments of Hoffman \& Amundsen [5] show that with a constant flow rate, the wax layer reaches an equilibrium height after sufficient time, with this time decreasing as the oil temperature increases. This basic feature is not predicted by the molecular diffusion model that is widely applied in the oil and gas industry [7,8] and a "shear-stripping" mechanism has to be introduced to match experimental observation with model predictions. A modelling approach based on the thermal phase change mechanism as outlined in [1] shows that this, and other fundamental experimental observations can be explained without the need to include rather speculative physical mechanisms. Very recently, the extensive review by Mehrotra et al. [9] has given a thorough consideration of experimental evidence, which provides significant and substantial support for the thermal phase change mechanism introduced in [1], as the principal and key mechanism in the process of wax deposition on the interior wall of pipes transporting heated oil. Most recently, a further review has been provided by Van der Geest et al. [10], which again supplies detailed and critical experimental support for the thermal phase change mechanism. In addition to these reviews, a recent thesis has addressed this issue experimentally, Mahir [4], and has also provided significant support for the thermal mechanism proposed in [1]. ${ }^{2}$ With the very recent emergence of this significant and compelling support for the basic thermal model introduced in [1], the purpose of the present paper is to investigate how the mathematical model in [1] can be further developed to accommodate more detailed features associated with wax phase change. Specifically, in this paper, we develop the model in [1] to account for the dependence of the thermal conductivity of the paraffinic wax solid on local temperature. This effect can be significant in many paraffinic wax materials when in solid crystalline state and is therefore an effect which should be investigated in the modelling process. Indeed, this feature of a temperature-dependent crystalline wax conductivity has been addressed experimentally in the recent paper by Viega et al. [11]. In the conclusion of [11] it is reported that up to a $30 \%$ drop in measured wax conductivity can be recorded through the wax layer, as the temperature drops from the wax appearance temperature, at the wax formation interface, to the temperature at the solid boundary of the pipe. This is attributed, in [11], to variations in the small liquid fraction captured in the crystallised wax structure. This paper certainly gives impetus to the study undertaken here. It specifically indicates that we may expect wax layer local conductivity to decrease as local temperature decreases, with as much as a $30 \%$ variation within a given wax layer. With this inclusion in the mathematical model developed in [1], the significant change is that the associated free boundary problem [IBVP] becomes non-linear. In particular, the partial differential equation, rather than being the associated linear heat conduction equation, becomes a non-linear (in fact uniformly quasi-linear) strictly parabolic equation, with, in addition, the mixed Robin-type linear boundary condition on the

\footnotetext{
1 Additionally see https://www.linkedin.com/company/tupdp/?feedView.

2 An interesting video of the experimental formation of a wax layer can be found at https://www.tupdp.org/? trk=organization-update_share-update_update-text, which also provides qualitative visual support for the wax thermal phase change mechanism.
} 
solid interior pipe wall, and the latent heat boundary condition on the free solid wax layer surface, both adopting a generalised non-linear form.

The principal aim of the current paper is to fully investigate the effects of the inclusion of the temperaturedependent solid wax thermal conductivity in the model developed in [1]. In Sect. 2, we review and extend the thermal phase change model and its mathematical formulation. This is followed in Sect. 3 by an extensive study of associated qualitative results for [IBVP]. These structural results are then complemented by consideration of the nature of the solution to [IBVP] as $t \rightarrow 0^{+}$and $t \rightarrow \infty$. In Sect. 5, we devote attention to developing a complete and tractable theory for [IBVP] when the parameter $\varepsilon$ is small, a case which often pertains in physical applications. In Sect. 6, we consider numerical solutions to [IBVP] for comparison with the theory developed earlier, whilst Sect. 7 gives a qualitative comparison with experiments presented in [5]. Finally, we end with a discussion in Sect. 8.

\section{The model}

Here, following [1], we develop the thermal phase change model which was introduced in [1], and discussed in Sect. 1. A schematic diagram of the model is displayed in Fig. 1. The heated oil is in uniform flow through a long, straight section of pipe, with circular cross section, and internal radius $R$. When the wax layer thickness on the interior pipe wall $h$ is very much smaller than the pipe radius $R(h \ll R)$, which is generally the case in applications (see, for example, the measurements reported in Halstensen et al. [6], where a typical pipe diameter is approximately $30 \mathrm{~mm}$ and wax layer thickness is approximately $1-2 \mathrm{~mm}$ ) then we can take full advantage of this in the model to reduce the spatial dimension to one. Following [1], we consider a long straight section of the pipe which is unaffected by the localised effects of fluid entering or leaving the pipe. Specifically, in this situation, we may suppose that all quantities of interest in the model are independent of the axial distance along the pipe. Therefore, the dependent variables in the model are functions of $x$ and $t$ only, with $x$ denoting the normal distance from the inner pipe wall towards the centre of the pipe, and with $t$ denoting time. The pipe is surrounded by an aligned circular coolant jacket of width $d_{\mathrm{c}}$. Moreover, the fluid in the coolant jacket is kept at a constant temperature $T_{\mathrm{c}}$. The thickness of the pipe wall is $d_{\mathrm{p}}$, with the outer pipe wall located at $x=-d_{\mathrm{p}}$ and the inner pipe wall located at $x=0$. The temperature within the pipe wall is denoted by $T_{\mathrm{p}}$. The solid wax layer is initiated, when $t=0$, at the inner pipe wall $x=0$, with its upper surface at $x=h$. The temperature within the solid wax layer is denoted by $T$, with $T_{h}$ being the constant temperature of the wax formation, at $x=h$. The experimental observations in [3] justify an approximation of the temperature of the oil flowing in the pipe, denoted by $T_{\mathrm{o}}$ for $h<x \leq R$, to be constant. Throughout, we consider the situation when

$T_{\mathrm{c}}<T_{h}<T_{\mathrm{o}}$.

We now consider the temperature field in the pipe wall. An application of Fourier's law gives

$T_{p t}=\frac{k_{\mathrm{p}}}{c_{\mathrm{p}} \rho_{\mathrm{p}}} T_{\mathrm{p} x x}, \quad-d_{\mathrm{p}}<x<0, t>0$,

where $k_{\mathrm{p}}, c_{\mathrm{p}}$ and $\rho_{\mathrm{p}}$ are the conductivity, specific heat capacity and density of the pipe wall, respectively. With $t_{\mathrm{S}}$ (as given in (12) and (18)) being the time scale associated with wax layer formation, the thickness of the pipe wall is thin, and such that

$d_{\mathrm{p}}^{2} \ll \frac{\left(k_{\mathrm{p}} t_{\mathrm{s}}\right)}{\left(c_{\mathrm{p}} \rho_{\mathrm{p}}\right)}$.

Consequently, the temperature in the pipe wall is in a quasi-steady state, and Eq. (2) may be approximated by

$T_{\mathrm{p} x x}=0 ; \quad-d_{\mathrm{p}}<x<0, \quad t \geq 0$,

subject to the following boundary conditions: 


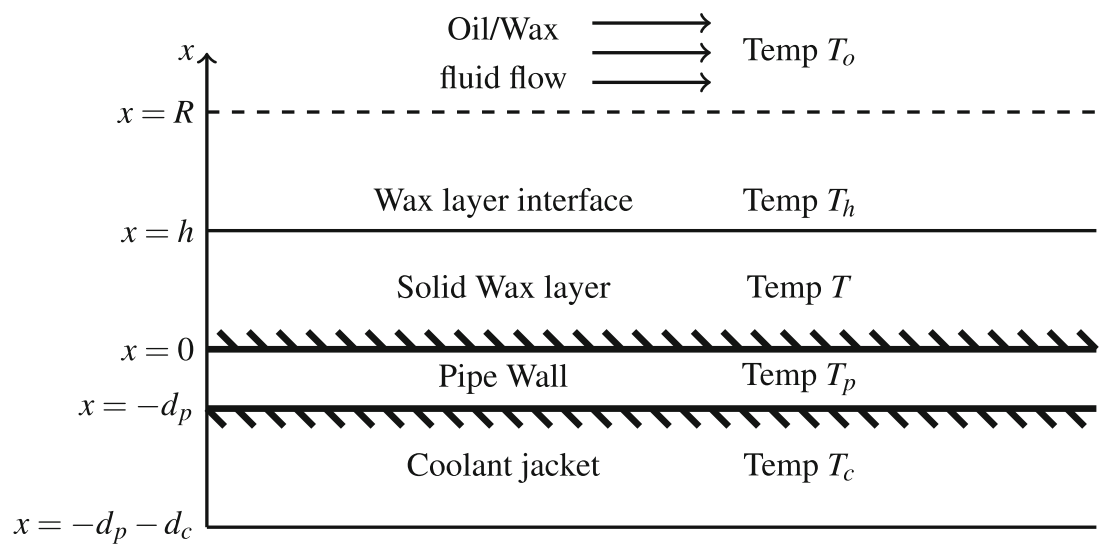

Fig. 1 Schematic diagram of the physical problem

$$
\begin{array}{ll}
k_{\mathrm{p}} T_{\mathrm{p} x}=\frac{k_{\mathrm{c}} N u_{\mathrm{c}}}{d_{\mathrm{c}}}\left(T_{\mathrm{p}}-T_{\mathrm{c}}\right) & x=-d_{\mathrm{p}}, \quad t \geq 0, \\
k_{\mathrm{p}} T_{\mathrm{p} x}=k_{\mathrm{W}}(T) T_{x} & x=0, \quad t \geq 0, \\
T_{\mathrm{p}}=T & x=0, \quad t \geq 0 .
\end{array}
$$

Here $k_{\mathrm{c}}$ is the coolant conductivity, $N u_{\mathrm{c}}$ is the Nusselt number for the coolant flow and $k_{\mathrm{w}}(T)$ is the temperaturedependent solid wax conductivity. Condition (4) represents continuity of heat flux across the exterior wall of the pipe, which is in contact with the coolant jacket, whilst condition (5) represents continuity of heat flux from the lower boundary of the wax layer into the interior pipe wall and condition (6) represents continuity of temperature at the lower boundary of the wax layer and the inner pipe wall. In allowing the solid wax conductivity to be temperature dependent, we write

$k_{\mathrm{w}}(T)=k_{\mathrm{w}}^{h} D\left(\frac{T-T_{\mathrm{c}}}{T_{h}-T_{\mathrm{c}}}\right)$

for $-\infty<T<\infty$, where $k_{\mathrm{w}}^{h}$ is the conductivity of the solid wax at the temperature of wax solidification $T=T_{h}$, and $D: \mathbb{R} \rightarrow \mathbb{R}$ is the dimensionless solid wax conductivity, representing the variation in solid wax conductivity with

$u=\frac{\left(T-T_{\mathrm{c}}\right)}{\left(T_{h}-T_{\mathrm{c}}\right)}$.

In general $D: \mathbb{R} \rightarrow \mathbb{R}$ will be taken as smooth, bounded and bounded above zero. Thus, throughout, we will consider $D: \mathbb{R} \rightarrow \mathbb{R}$ to satisfy the conditions:

(D1) $D(1)=1$,

(D2) $D_{m} \leq D(u) \leq D_{M}$ for all $u \in \mathbb{R}$, with $D_{m}$ and $D_{M}$ being positive constants,

(D3) $D \in C^{3}(\mathbb{R})$.

Although conditions (D1)-(D3) are sufficient to develop a theory for the model under examination here, we note that the conclusions section of [11], after a programme of careful experiments, highlight that $D(u)$ should also be an increasing function for $u \in[0,1]$, and that $D(u)$ evaluated at the pipe wall should be generally about $30 \%$ less than $D(u)$ evaluated at the wax formation interface. Now, the solution to (3), (4) and (6) is given by

$T_{\mathrm{p}}(x, t)=\frac{k_{\mathrm{c}} N u_{\mathrm{c}}\left(T(0, t)-T_{\mathrm{c}}\right)}{\left(k_{\mathrm{p}} d_{\mathrm{c}}+k_{\mathrm{c}} N u_{\mathrm{c}} d_{\mathrm{p}}\right)} x+T(0, t) ; \quad-d_{\mathrm{p}} \leq x \leq 0, \quad t \geq 0$,

after which boundary condition (5) then requires

$k_{\mathrm{w}}(T) T_{x}=\frac{k_{\mathrm{p}} k_{\mathrm{c}} N u_{\mathrm{c}}}{\left(k_{\mathrm{p}} d_{\mathrm{c}}+k_{\mathrm{c}} N u_{\mathrm{c}} d_{\mathrm{p}}\right)}\left(T-T_{\mathrm{c}}\right), \quad x=0, \quad t>0$, 
which is a condition on $T$ at $x=0$, the lower boundary of the solid wax layer at the inner pipe wall. We are now in a position to consider the temperature field $T$ in the solid wax layer. Fourier's law requires that

$\rho_{\mathrm{W}} c_{\mathrm{w}} T_{t}=\left(k_{\mathrm{w}}(T) T_{x}\right)_{x} \quad 0<x<h(t), \quad t>0$,

subject to the boundary conditions

$\begin{array}{ll}k_{\mathrm{w}}(T) T_{x}=\frac{k_{\mathrm{p}} k_{\mathrm{c}} N u_{\mathrm{c}}}{\left(k_{\mathrm{p}} d_{\mathrm{c}}+k_{\mathrm{c}} N u_{\mathrm{c}} d_{\mathrm{p}}\right)}\left(T-T_{\mathrm{c}}\right) & x=0, \quad t>0, \\ T=T_{h} & x=h(t), \quad t>0, \\ \rho_{\mathrm{w}} H_{\mathrm{w}} h_{t}=k_{\mathrm{w}}(T) T_{x}-\frac{k_{\mathrm{o}} N u}{R}\left(T_{\mathrm{o}}-T_{h}\right) & x=h(t), \quad t>0,\end{array}$

where $\rho_{\mathrm{w}}, c_{\mathrm{w}}$ and $H_{\mathrm{w}}$ are the density, specific heat capacity and latent heat of the solid wax, respectively, whilst $k_{\mathrm{o}}$ and $\mathrm{Nu}$ are the conductivity of oil and the Nusselt number for the oil flow, respectively. The conditions (9) and (10) express that the outer surface of the solid wax layer must be at the wax solidification temperature, and that the difference in heat $f l u x$ across this interface balances the latent heat required for solid wax formation. For convenience, we non-dimensionalised the free boundary problem for $T(x, t)$ and $h(t)$ given by (7)-(10). We introduce the dimensionless variables

$u=\frac{T-T_{\mathrm{c}}}{T_{\mathrm{s}}}, \quad x^{\prime}=\frac{x}{x_{\mathrm{s}}}, \quad t^{\prime}=\frac{t}{t_{\mathrm{s}}}, \quad h^{\prime}=\frac{h}{x_{\mathrm{s}}}$

with the scales $T_{\mathrm{s}}, x_{\mathrm{s}}$ and $t_{\mathrm{s}}$ chosen as

$T_{\mathrm{s}}=T_{h}-T_{\mathrm{c}}, \quad x_{\mathrm{s}}=\frac{R k_{\mathrm{w}}^{h}\left(T_{h}-T_{\mathrm{c}}\right)}{k_{\mathrm{o}} N u\left(T_{\mathrm{o}}-T_{h}\right)}, \quad t_{\mathrm{s}}=\frac{R^{2} \rho_{\mathrm{w}} H_{\mathrm{w}} k_{\mathrm{w}}^{h}\left(T_{h}-T_{\mathrm{c}}\right)}{k_{\mathrm{o}}^{2} N u^{2}\left(T_{\mathrm{o}}-T_{h}\right)^{2}}$.

On substituting (11) and (12) into (7)-(10), we obtain the non-dimensional form of the free boundary problem as

$\varepsilon u_{t}=\left(D(u) u_{x}\right)_{x} \quad 0<x<h(t), \quad t>0$,

$D(u) u_{x}=k u \quad x=0, t>0$,

$u=1 \quad x=h(t), \quad t>0$,

$h_{t}=D(u) u_{x}-1 \quad x=h(t), \quad t>0$,

where the two dimensionless parameters $\varepsilon$ and $k$ are given by

$\varepsilon=\frac{c_{\mathrm{W}}\left(T_{h}-T_{\mathrm{c}}\right)}{H_{\mathrm{w}}}, \quad k=\frac{k_{\mathrm{p}} k_{\mathrm{c}} N u_{\mathrm{c}} R\left(T_{h}-T_{\mathrm{c}}\right)}{k_{\mathrm{o}} N u\left(k_{\mathrm{p}} d_{\mathrm{c}}+k_{\mathrm{c}} N u_{\mathrm{c}} d_{\mathrm{p}}\right)\left(T_{\mathrm{o}}-T_{h}\right)}$.

Here primes have been dropped for ease of notation. The parameter $\varepsilon$ is a dimensionless solid wax conductivity number, and measures the ratio of the time scale for heat conduction in the wax layer to the time scale for wax layer growth, whilst the parameter $k$ is a dimensionless boundary cooling number, and measures the ratio of heat extracted from the wax layer, by cooling, to the heat the wax layer gains from the oil. This is a free boundary problem for $u(x, t)$ with $0 \leq x \leq h(t), t>0$ and $h(t) \geq 0$. It is worth noting that typical values, estimated in Schulkes [2] and Kaye and Laby [12], for the scales in (12), are

$T_{\mathrm{S}} \sim 10^{\circ} \mathrm{C}, \quad x_{\mathrm{s}} \sim 0.16 \mathrm{~m}, \quad t_{\mathrm{s}} \sim 2 \times 10^{6} \mathrm{~s} \sim 20$ days

with the dimensionless parameters

$\varepsilon \sim O\left(10^{-1}\right), \quad k \sim O\left(\frac{2 N u_{\mathrm{c}}}{1+10^{-3} N u_{\mathrm{c}}}\right)$.

We observe from (17) that the parameter $\varepsilon$ is independent of both the core oil flow Nusselt number $N u$ and the coolant flow Nusselt number $N u_{\mathrm{c}}$. However, the parameter $k$ decreases with the increase in oil Nusselt number, whilst it increases with the increase in coolant Nusselt number, with the limiting value being

$k \sim \frac{k_{\mathrm{p}} R\left(T_{h}-T_{\mathrm{c}}\right)}{k_{\mathrm{o}} N u d_{\mathrm{p}}\left(T_{\mathrm{o}}-T_{h}\right)}$ 
for large $N u_{\mathrm{c}}$.

\section{The free boundary problem [IBVP]}

The free boundary problem associated with the mathematical model introduced in Sect. 2 ((13)-(16)) may be written fully as

$$
\begin{array}{ll}
\varepsilon u_{t}=\left(D(u) u_{x}\right)_{x} & 0<x<h(t), \quad t>0, \\
D(u) u_{x}=k u & x=0, \quad t>0, \\
u=1 & x=h(t), \quad t>0, \\
h_{t}=D(u) u_{x}-1 & x=h(t), \quad t>0,
\end{array}
$$

The problem, (19)-(24), will be referred to as [IBVP]. For any $T>0$, the following subsets of $\mathbb{R}^{2}$ are also introduced, namely,

$$
\begin{aligned}
& D_{T}=\left\{(x, t) \in \mathbb{R}^{2}: 0<x<h(t), 0<t \leq T\right\}, \\
& \partial D_{L}^{\mathrm{T}}=\left\{(x, t) \in \mathbb{R}^{2}: x=0,0<t \leq T\right\}, \\
& \partial D_{R}^{\mathrm{T}}=\left\{(x, t) \in \mathbb{R}^{2}: x=h(t), 0<t \leq T\right\}, \\
& \partial D_{T}=\partial D_{L}^{\mathrm{T}} \cup \partial D_{R}^{\mathrm{T}},
\end{aligned}
$$

with closures denoted by $\bar{D}_{T}, \partial \bar{D}_{L}^{\mathrm{T}}, \partial \bar{D}_{R}^{\mathrm{T}}$ and $\partial \bar{D}_{T}$. A solution to [IBVP] will be considered as classical, with the following regularity requirements,

(R1) $h:[0, \infty) \rightarrow \mathbb{R}$ is continuous and the derivative $h_{t}$ exists and is continuous on $(0, \infty)$, with $h$ and $h_{t}$ non-negative on $(0, \infty)$.

(R2) $u: \bar{D}_{\infty} \rightarrow \mathbb{R}$ is continuous and $u_{x}, u_{t}$ both exist and are continuous on $\bar{D}_{\infty}$ and $u_{x x}$ exists and is continuous on $D_{\infty}$.

A reformulation of [IBVP] (with (R1) and (R2)) in terms of coupled integral equations is given by Friedman [13] and used by Schatz [14] and Cannon and Hill [15] to study the regularity of solutions to [IBVP]. It is established by Cannon and Hill [15] that with $u: \bar{D}_{\infty} \rightarrow \mathbb{R}$ and $h:[0, \infty) \rightarrow \mathbb{R}$ being a solution to [IBVP] (with (R1) and (R2)) then, in fact,

$u \in C^{3}\left(\bar{D}_{\infty}\right)$ and $h \in C^{1}([0, \infty))$,

which requires (D3), in particular. A consequence of (R1) and (25) is also

$h(t) \geq 0 \quad$ and $\quad h_{t}(t) \geq 0 \quad \forall t \in[0, \infty)$.

Before proceeding to analyse [IBVP] further, we first consider steady state solutions associated with [IBVP].

\subsection{Steady state solutions to [IBVP]}

A steady state solution to [IBVP] is a solution to [IBVP] which is independent of $t$. Thus, $u_{\mathrm{S}}:\left[0, h_{\mathrm{S}}\right] \rightarrow \mathbb{R}$ and $h_{\mathrm{s}}>0$ is a steady solution to [IBVP] whenever $u_{\mathrm{s}} \in C^{1}\left(\left[0, h_{\mathrm{s}}\right]\right) \cap C^{2}\left(\left(0, h_{\mathrm{s}}\right)\right)$, and satisfies the boundary value problem 
$\left(D\left(u_{\mathrm{s}}\right) u_{\mathrm{s} x}\right)_{x}=0 \quad 0<x<h_{\mathrm{s}}$,

$D\left(u_{\mathrm{s}}\right) u_{\mathrm{s} x}=k u_{\mathrm{s}} \quad x=0$,

$u_{\mathrm{s}}=1 \quad x=h_{\mathrm{s}}$,

$D\left(u_{\mathrm{s}}\right) u_{\mathrm{s} x}=1 \quad x=h_{\mathrm{s}}$.

It is now convenient to introduce $F: \mathbb{R} \rightarrow \mathbb{R}$ given by

$F(X)=\int_{0}^{X} D(\lambda) \mathrm{d} \lambda \quad \forall X \in \mathbb{R}$,

where $F(0)=0$ and

$F^{\prime}(X)=D(X)>0 \quad \forall X \in \mathbb{R}$.

Observe, via (D2) and (D3), that $F(X)$ is strictly increasing with $X$, and $F \in C^{4}(\mathbb{R})$. Therefore, the inverse $F^{-1}: \mathbb{R} \rightarrow \mathbb{R}$ exists, with $F^{-1} \in C^{4}(\mathbb{R})$, via (32). The boundary value problem (27)-(30) can now be written as

$$
\begin{array}{ll}
\left(F\left(u_{\mathrm{s}}\right)\right)_{x x}=0 & 0<x<h_{\mathrm{s}}, \\
\left(F\left(u_{\mathrm{s}}\right)\right)_{x}=k u_{\mathrm{s}} & x=0, \\
u_{\mathrm{s}}=1 & x=h_{\mathrm{s}}, \\
\left(F\left(u_{\mathrm{s}}\right)\right)_{x}=1 & x=h_{\mathrm{s}} .
\end{array}
$$

An integration of (33) gives

$F\left(u_{\mathrm{s}}(x)\right)=A x+B \quad 0 \leq x \leq h_{\mathrm{s}}$,

where $A, B \in \mathbb{R}$ are constants. Applying (34) and (36), we obtain

$A=1 \quad$ and $B=F\left(k^{-1}\right)$,

after which, (37) becomes

$F\left(u_{\mathrm{s}}(x)\right)=x+F\left(k^{-1}\right) ; \quad 0 \leq x \leq h_{\mathrm{s}}$.

Finally, applying (35) and rearranging, we require

$h_{\mathrm{s}}=F(1)-F\left(k^{-1}\right)$.

In a steady state we must have $h_{\mathrm{s}}>0$. Therefore, it follows from (39) and (40) that we have established:

Proposition 1 A steady state solution to [IBVP] exists, and is unique, if and only if, $k>1$. The steady state solution is given by (39) with (40).

It is worth noting that (40) may be written as

$h_{\mathrm{s}}=\left(1-k^{-1}\right)\left\langle D\left(\left[k^{-1}, 1\right]\right)\right\rangle$,

where

$\left\langle D\left(\left[k^{-1}, 1\right]\right)\right\rangle=\left(1-k^{-1}\right)^{-1} \int_{k^{-1}}^{1} D(\lambda) \mathrm{d} \lambda$

is the mean value of $D(\lambda)$ over the interval $\lambda \in\left[k^{-1}, 1\right]$. Also, we may rewrite (39) explicitly as

$u_{\mathrm{S}}(x)=F^{-1}\left(x+F\left(k^{-1}\right)\right) \quad 0 \leq x \leq h_{\mathrm{s}}$.

An examination of $h_{\mathrm{S}}=h_{s}(k)$ in (41) establishes the following properties: 


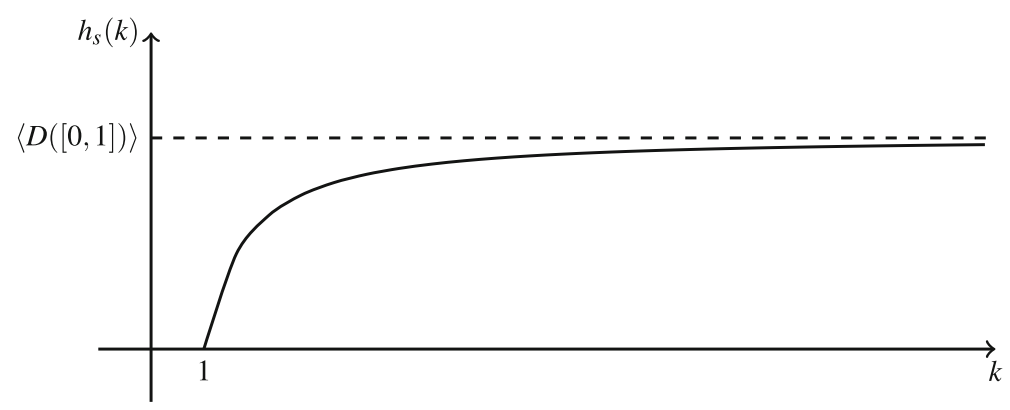

Fig. 2 Sketch of $h_{\mathrm{s}}(k)$ against $k$
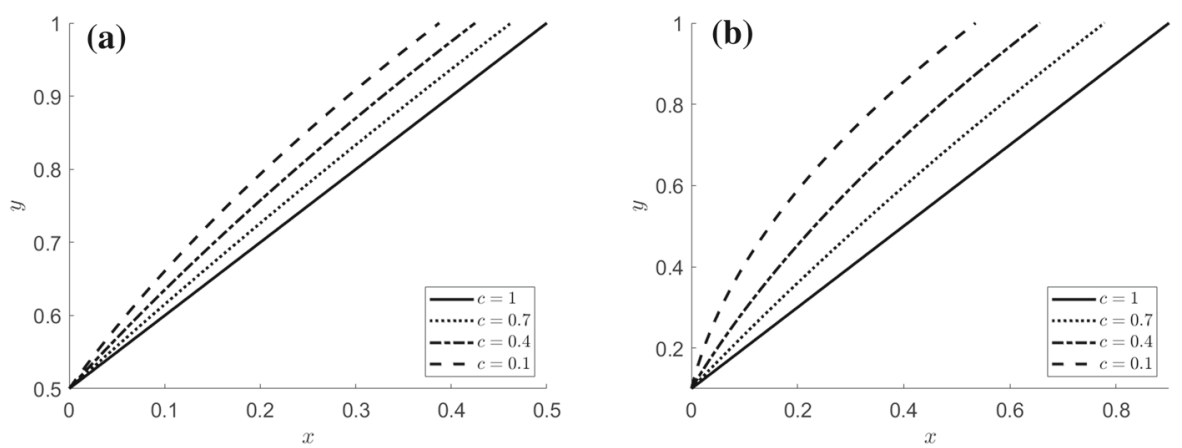

Fig. 3 Plots of $y=u_{\mathrm{s}}(x)$ for $D(u)=c+(1-c) u$ with $k=2$ and 10 in a and $\mathrm{b}$, respectively

(i) $h_{\mathrm{s}} \in C^{4}([1, \infty))$ and is strictly monotone increasing,

(ii) $h_{\mathrm{s}}^{\prime}(k)=D\left(k^{-1}\right) k^{-2} \quad \forall k \in[1, \infty)$,

(iii) $h_{\mathrm{s}}(k)=(k-1)+O\left((k-1)^{2}\right)$ as $k \rightarrow 1^{+}$,

(iv) $h_{\mathrm{s}}(k)=\langle D([0,1])\rangle-D(0) k^{-1}+O\left(k^{-2}\right)$ as $k \rightarrow \infty$.

We give a qualitative sketch of $h_{\mathrm{S}}(k)$ against $k$ in Fig. 2. Next, an examination of (43) establishes the following properties of $u_{\mathrm{s}}:\left[0, h_{\mathrm{s}}\right] \rightarrow \mathbb{R}:$

(i) $u_{\mathrm{s}} \in C^{4}\left(\left[0, h_{\mathrm{s}}\right]\right)$ and is monotone increasing,

(ii) $u_{\mathrm{s}}(0)=k^{-1}$ and $u_{\mathrm{s}}\left(h_{\mathrm{s}}\right)=1$,

(iii) $u_{\mathrm{s}}^{\prime}(x)=\frac{1}{D\left(u_{\mathrm{s}}(x)\right)}>0 \quad \forall x \in\left[0, h_{\mathrm{s}}\right]$,

(iv) $u_{\mathrm{s}}^{\prime \prime}(x)=-\frac{D^{\prime}\left(u_{\mathrm{s}}(x)\right)}{D\left(u_{\mathrm{s}}(x)\right)^{3}} \quad \forall x \in\left[0, h_{\mathrm{s}}\right]$.

For illustrative purposes, steady state solutions associated with specific crystallised wax conductivities given by $D(u)=c+(1-c) u$ for all $u \in[0,1]$ with values of $c=1.0,0.7,0.4$ and 0.1 are shown in Fig. 3a-b. This form is taken so as to conform with the experimental measurements reported by Viega et al. in [11]. The values of $c=0.4$ and $c=0.7$ are chosen to reproduce a reduction in wax conductivity of order of $30 \%$ across the wax layer in Fig. $3 \mathrm{a}$ and $b$, respectively, in accord with [11]. We see, in Fig. 3a-b, that the effect of making the conductivity variation stronger is to lower the temperature profile across the crystallised wax layer. Each temperature profile, apart from that with constant conductivity $(c=1)$, has a weak negative curvature. This is in very good agreement with the temperature profiles measured experimentally in [11]. Indeed, a comparison between the theoretical profiles, shown in Fig. 3a-b, and the experimental profiles, reported in [11, Fig. 12], shows remarkable agreement which is very encouraging for the present theory. We now return to [IBVP]. 


\subsection{Qualitative theory for [IBVP]}

In this subsection, we determine the principal structure and qualitative properties of the solution to the free boundary problem [IBVP]. To begin with, we have:

Proposition 2 Let $u: \bar{D}_{\infty} \rightarrow \mathbb{R}$ be a solution to $[I B V P]$. Then

$0 \leq u(x, t) \leq 1 \quad \forall(x, t) \in \bar{D}_{\infty}$.

Proof Consider $u: \bar{D}_{\infty} \rightarrow \mathbb{R}$ on the compact region $\bar{D}_{T}$ (for any $T>0$ ). Then $u$ is continuous on $\bar{D}_{T}$ since $u$ is continuous on $\bar{D}_{\infty}$ (via (R2)). Also, we have that $u_{x}, u_{t}$ and $u_{x x}$ all exist and are continuous on $D_{\infty}$ and so are continuous on $D_{T}$ (via (R2)). Also, we have from (19) that

$\varepsilon u_{t}=\left(D(u) u_{x}\right)_{x}$ on $D_{\infty}$,

and so,

$u_{t}-\varepsilon^{-1} D^{\prime}(u) u_{x}{ }^{2}-\varepsilon^{-1} D(u) u_{x x}=0 \quad$ on $D_{\infty}$.

Now for each $(x, t) \in D_{T}$, set

$a(x, t)=\varepsilon^{-1} D(u) \geq \varepsilon^{-1} D_{m}>0$,

via (D2) and

$b(x, t)=\varepsilon^{-1} D^{\prime}(u) u_{x}$.

Now suppose that $u$ is not non-negative on $\bar{D}_{T}$. Then using (44)-(46), we may apply the Weak Parabolic Minimum Principle [16] to conclude that there exists a point $\left(x^{*}, t^{*}\right) \in \partial \bar{D}_{T}$ such that

$u\left(x^{*}, t^{*}\right)=\inf _{(x, t) \in \bar{D}_{T}} u(x, t)<0$.

Since $\left(x^{*}, t^{*}\right) \in \partial \bar{D}_{T}=\partial \bar{D}_{L}^{\mathrm{T}} \cup \partial \bar{D}_{R}^{\mathrm{T}}$ we must have $\left(x^{*}, t^{*}\right)=\left(0, t^{*}\right) \in \partial \bar{D}_{L}^{\mathrm{T}}$ otherwise $u\left(x^{*}, t^{*}\right)=1$. Thus, at $\left(0, t^{*}\right)$ we have

$D\left(u\left(0, t^{*}\right)\right) u_{x}\left(0, t^{*}\right)=k u\left(0, t^{*}\right)<0$,

via condition (20) and (47). Then, via (D2), we conclude that

$u_{x}\left(0, t^{*}\right)<0$.

However, since $u$ achieves its infimum at $\left(0, t^{*}\right)$ then $u_{x}\left(0, t^{*}\right) \geq 0$, which contradicts (48). Thus, we conclude that $u$ must be non-negative on $\bar{D}_{T}$. This holds for any $T>0$ and so $u(x, t) \geq 0$ for all $(x, t) \in \bar{D}_{\infty}$. Next it follows from (21) that

$$
\sup _{(x, t) \in \bar{D}_{T}} u(x, t) \geq 1 .
$$

However, via (44)-(46), it follows from the Weak Parabolic Maximum Principle [16] that there exists a point $\left(x^{*}, t^{*}\right) \in \partial \bar{D}_{T}$ such that

$u\left(x^{*}, t^{*}\right)=\sup _{(x, t) \in \bar{D}_{T}} u(x, t) \geq 1$

via (49). Now suppose that $\left(x^{*}, t^{*}\right) \in \partial \bar{D}_{L}^{\mathrm{T}}$, so that $\left(x^{*}, t^{*}\right)=\left(0, t^{*}\right)$, and from (20) and (50) we have that $D\left(u\left(0, t^{*}\right)\right) u_{x}\left(0, t^{*}\right)=k u\left(0, t^{*}\right) \geq k>0$ 
and so,

$u_{x}\left(0, t^{*}\right)>0$

via (D2). However, since $u$ achieves its supremum at $\left(0, t^{*}\right)$ then $u_{x}\left(0, t^{*}\right) \leq 0$, which contradicts (51). Hence we conclude that $\left(x^{*}, t^{*}\right) \in \partial \bar{D}_{R}^{\mathrm{T}}$, and so, via (21),

$u\left(x^{*}, t^{*}\right)=\sup _{(x, t) \in \bar{D}_{T}} u(x, t)=1$.

Therefore, $u \leq 1$ on $\bar{D}_{T}$. Since this holds for any $T>0$, we conclude that $u(x, t) \leq 1$ for all $(x, t) \in \bar{D}_{\infty}$. Consequently, we have

$0 \leq u(x, t) \leq 1 \quad \forall(x, t) \in \bar{D}_{\infty}$,

as required.

We next refine these inequalities in

Corollary 1 Let $u: \bar{D}_{\infty} \rightarrow \mathbb{R}$ be a solution to [IBVP]. Then

$0<u(x, t)<1 \quad \forall(x, t) \in D_{\infty}$.

Proof From Proposition 2 we have

$0 \leq u(x, t) \leq 1 \quad \forall(x, t) \in \bar{D}_{\infty}$.

Now suppose there exists a point $\left(x^{*}, t^{*}\right) \in D_{\infty}$ such that $u\left(x^{*}, t^{*}\right)=0$. Then

$\inf _{(x, t) \in \bar{D}_{\infty}} u(x, t)=0$

and it follows from the Strong Parabolic Minimum Principle [16] that

$u(x, t)=0 \quad \forall(x, t) \in \bar{D}_{T^{*}}$,

where

$T^{*}=\sup \{t \in(0, T]: \exists x \in(0, h(t))$ with $u(x, t)=0\} \geq t^{*}>0$.

This contradicts condition (21). Thus, $u(x, t)>0$ for all $(x, t) \in D_{\infty}$. Next suppose that there exists $\left(x^{*}, t^{*}\right) \in D_{\infty}$ such that $u\left(x^{*}, t^{*}\right)=1$. Then

$$
\sup _{(x, t) \in \bar{D}_{\infty}} u(x, t)=1,
$$

and it follows from the Strong Parabolic Maximum Principle [16] that

$u(x, t)=1 \quad \forall(x, t) \in \bar{D}_{T^{*}}$,

where now

$T^{*}=\sup \{t \in(0, T]: \exists x \in(0, h(t))$ with $u(x, t)=1\} \geq t^{*}>0$.

Also, via condition (20) with regularity condition (R2), we have from (52),

$D(u(0, t)) u_{x}(0, t)=k u(0, t)=k>0 \quad \forall t \in\left[0, T^{*}\right]$,

and so, via (D2),

$u_{x}(0, t)>0 \quad \forall t \in\left[0, T^{*}\right]$.

However, since $u(x, t)=1$ for all $(x, t) \in \bar{D}_{T^{*}}$, then $u_{x}(0, t)=0$ for all $t \in\left[0, T^{*}\right]$, which contradicts (53). Thus $u(x, t)<1$ for all $(x, t) \in D_{\infty}$. Therefore,

$0<u(x, t)<1 \quad \forall(x, t) \in D_{\infty}$,

as required. 
Next we have

Proposition 3 Let $u: \bar{D}_{\infty} \rightarrow \mathbb{R}$ be a solution to $[I B V P]$. Then

$u_{t}(x, t) \leq 0 \quad \forall(x, t) \in \bar{D}_{\infty}$.

Proof Firstly, from (19) we have

$\varepsilon D(u) u_{t}=D(u)\left(D(u) u_{x}\right)_{x} \quad$ on $D_{\infty}$.

Next we introduce $f: \bar{D}_{\infty} \rightarrow \mathbb{R}$ defined by

$f(x, t)=F(u(x, t)) \quad \forall(x, t) \in \bar{D}_{\infty}$,

with $F: \mathbb{R} \rightarrow \mathbb{R}$ as introduced in (31). It follows from (31), (25) and (D3) that

$f \in C^{3}\left(\bar{D}_{\infty}\right)$,

and we have, for $(x, t) \in \bar{D}_{\infty}$,

$f_{t}(x, t)=F^{\prime}(u(x, t)) u_{t}(x, t)=D(u(x, t)) u_{t}(x, t)$,

$f_{x}(x, t)=F^{\prime}(u(x, t)) u_{x}(x, t)=D(u(x, t)) u_{x}(x, t)$,

$f_{x x}(x, t)=D^{\prime}(u(x, t)) u_{x}^{2}(x, t)+D(u(x, t)) u_{x x}(x, t)$.

Therefore, via (57) and (58), we observe that (54) becomes

$\varepsilon f_{t}=D(u) f_{x x} \quad$ on $D_{\infty}$.

Similarly, (20)-(22) becomes

$f_{x}=k u \quad x=0, \quad t>0$,

$f=F(1) \quad x=h(t), \quad t>0$,

$h_{t}=f_{x}-1 \quad x=h(t), \quad t>0$.

We next introduce $w: \bar{D}_{\infty} \rightarrow \mathbb{R}$ defined by

$w(x, t)=f_{t}(x, t) \quad \forall(x, t) \in \bar{D}_{\infty}$.

From (56) it follows that

$w \in C^{2}\left(\bar{D}_{\infty}\right)$,

whilst from (56) and (60), we have that

$\varepsilon f_{t t}=D(u) f_{x x t}+D^{\prime}(u) u_{t} f_{x x}$ on $D_{\infty}$,

which becomes, via (57) and (64),

$\varepsilon w_{t}=D(u) w_{x x}+\frac{D^{\prime}(u)}{D(u)} f_{x x} w \quad$ on $D_{\infty}$.

Hence, we have

$w_{t}-a(x, t) w_{x x}-c(x, t) w=0 \quad$ on $D_{\infty}$,

where

$a(x, t)=\frac{D(u(x, t))}{\varepsilon}>\frac{D_{m}}{\varepsilon}>0 \quad \forall(x, t) \in \bar{D}_{\infty}$, 
via (D2), and

$c(x, t)=\frac{D^{\prime}(u(x, t))}{\varepsilon D(u(x, t))} f_{x x}(x, t) \quad \forall(x, t) \in \bar{D}_{\infty}$.

It follows from (25), (D3) and (56) that $c(x, t)$ is continuous on $\bar{D}_{\infty}$, and so is continuous and bounded on $\bar{D}_{T}$, for any $T>0$. Also, via (61) together with (25) and (56),

$f_{x t}=k u_{t} \quad x=0, \quad t>0$,

and so, via (57) and (64),

$w_{x}=\frac{k w}{D(u)} \quad x=0, \quad t>0$.

In addition, via (56), (62), regularity condition (R1) and the chain rule we have

$f_{t}+h_{t} f_{x}=0 \quad x=h(t), \quad t>0$,

and so, via (64),

$w=-h_{t} f_{x} \quad x=h(t), \quad t>0$.

However, regularity condition (R1) requires $h_{t} \geq 0$, and so, via (63),

$f_{x} \geq 1 \quad x=h(t), \quad t>0$.

Thus, we conclude from regularity condition (R1) and (69) that

$w \leq 0 \quad x=h(t), \quad t>0$.

Next we introduce $v: \bar{D}_{\infty} \rightarrow \mathbb{R}$ such that

$w(x, t)=\mathrm{e}^{\lambda t} v(x, t) \quad \forall(x, t) \in \bar{D}_{\infty}$,

with $\lambda \in \mathbb{R}$ to be chosen. From (65) it follows that

$v \in C^{2}\left(\bar{D}_{\infty}\right)$.

Thus, for $(x, t) \in \bar{D}_{\infty}$ we have

$w_{x}(x, t)=\mathrm{e}^{\lambda t} v_{x}(x, t)$,

$w_{t}(x, t)=\lambda \mathrm{e}^{\lambda t} v(x, t)+\mathrm{e}^{\lambda t} v_{t}(x, t)$,

$w_{x x}(x, t)=\mathrm{e}^{\lambda t} v_{x x}(x, t)$.

Then, via (71) and (72), with (66), we obtain

$v_{t}-a(x, t) v_{x x}-(c(x, t)-\lambda) v=0$ on $D_{\infty}$.

Now set $T>0$ and let $C_{T}>0$ be a bound for $c$ on $\bar{D}_{T}$, so that

$|c(x, t)| \leq C_{T} \quad \forall(x, t) \in \bar{D}_{T}$.

We next choose

$\lambda=2 C_{T}>0$

so that

$c(x, t)-\lambda \leq-C_{T}<0 \quad \forall(x, t) \in \bar{D}_{T}$. 
Suppose that $v$ is not non-positive on $\bar{D}_{T}$. Then

$\sup v(x, t)>0$,

$(x, t) \in \bar{D}_{T}$

and, via (70) and (71), this cannot be achieved on $\partial \bar{D}_{R}^{\mathrm{T}}$. Thus, there exists

$\left(x^{*}, t^{*}\right) \in D_{T} \cup \partial D_{L}^{\mathrm{T}}$,

such that

$v\left(x^{*}, t^{*}\right)=\sup _{(x, t) \in \bar{D}_{T}} v(x, t)>0$.

If $\left(x^{*}, t^{*}\right) \in D_{T}$ then $t^{*} \in(0, T)$ or $t^{*}=T$. When $t^{*} \in(0, T)$ then $\left(x^{*}, t^{*}\right)$ is such that

$v_{t}\left(x^{*}, t^{*}\right)=v_{x}\left(x^{*}, t^{*}\right)=0$,

$v_{x x}\left(x^{*}, t^{*}\right) \leq 0$.

However, from (76) with (67), (79), (81) and (83), we have

$v_{t}\left(x^{*}, t^{*}\right)=a\left(x^{*}, t^{*}\right) v_{x x}\left(x^{*}, t^{*}\right)+\left(c\left(x^{*}, t^{*}\right)-\lambda\right) v\left(x^{*}, t^{*}\right)<0$,

which contradicts (82). Similarly, when $t^{*}=T$, then $\left(x^{*}, T\right)$ is such that

$v_{x}\left(x^{*}, T\right)=0, \quad v_{x x}\left(x^{*}, T\right) \leq 0$,

and

$v_{t}\left(x^{*}, T\right) \geq 0$.

However, via (76) with (67), (79), (81) and (84), we have

$v_{t}\left(x^{*}, T\right)=a\left(x^{*}, T\right) v_{x x}\left(x^{*}, T\right)+\left(c\left(x^{*}, T\right)-\lambda\right) v\left(x^{*}, T\right)<0$,

which contradicts (85). Hence, we conclude that $\left(x^{*}, t^{*}\right) \notin D_{T}$, so that, from (80),

$\left(x^{*}, t^{*}\right)=\left(0, t^{*}\right) \in \partial D_{L}^{\mathrm{T}}$,

and, via (81),

$v_{x}\left(0, t^{*}\right) \leq 0$.

However, via (68), (71) and (73), we have

$v_{x}\left(0, t^{*}\right)=\frac{k v\left(0, t^{*}\right)}{D\left(u\left(0, t^{*}\right)\right)}>0$,

using (D2) and (81), which contradicts (86). Thus, we concluded that

$\left(x^{*}, t^{*}\right) \notin D_{T} \cup \partial D_{L}^{\mathrm{T}}$,

which contradicts (80). Hence, $v$ must be non-positive on $\bar{D}_{T}$, so that

$v(x, t) \leq 0 \quad \forall(x, t) \in \bar{D}_{T}$.

It then follows, via (64) and (71), that

$f_{t}(x, t) \leq 0 \quad \forall(x, t) \in \bar{D}_{T}$.

Therefore, from (57), we have

$u_{t}(x, t) \leq 0 \quad \forall(x, t) \in \bar{D}_{T}$.

Since this holds for any $T>0$ we have

$u_{t}(x, t) \leq 0 \quad \forall(x, t) \in \bar{D}_{\infty}$

as required. 
As a consequence, we have the refinement,

Corollary 2 Let $u: \bar{D}_{\infty} \rightarrow \mathbb{R}$ be a solution to $[I B V P]$. Then

$u_{t}(x, t)<0 \quad \forall(x, t) \in D_{\infty}$.

Proof We introduce the function $\bar{v}: \bar{D}_{\infty} \rightarrow \mathbb{R}$ such that

$v(x, t)=e^{\mu t} \bar{v}(x, t) \quad \forall(x, t) \in \bar{D}_{\infty}$,

with $v: \bar{D}_{\infty} \rightarrow \mathbb{R}$ as introduced in (71) and with $\mu \in \mathbb{R}$ to be chosen. From (72) it follows that

$\bar{v} \in C^{2}\left(\bar{D}_{\infty}\right)$.

whilst from (87),

$\bar{v}(x, t) \leq 0 \quad \forall(x, t) \in \bar{D}_{\infty}$.

From (88) we have that for $(x, t) \in \bar{D}_{\infty}$,

$v_{x}(x, t)=\mathrm{e}^{\mu t} \bar{v}_{x}(x, t)$,

$v_{t}(x, t)=\mu \mathrm{e}^{\mu t} \bar{v}(x, t)+\mathrm{e}^{\mu t} \bar{v}_{t}(x, t)$,

$v_{x x}(x, t)=\mathrm{e}^{\mu t} \bar{v}_{x x}(x, t)$.

Hence, after substituting (91)-(93) into (76), we obtain

$\bar{v}_{t}-a(x, t) \bar{v}_{x x}-(c(x, t)-(\lambda+\mu)) \bar{v}=0 \quad \forall(x, t) \in D_{\infty}$.

Setting $T>0$ and recalling (77) and (78), we have

$c(x, t)-(\lambda+\mu) \geq-C_{T}-\left(2 C_{T}+\mu\right)=-3 C_{T}-\mu \quad \forall(x, t) \in \bar{D}_{T}$.

We now choose

$\mu=-3 C_{T}$,

so that

$c(x, t)-(\lambda+\mu) \geq 0 \quad \forall(x, t) \in \bar{D}_{T}$.

Thus, from (94) and (95) together with (90), we have

$\bar{v}_{t}-a(x, t) \bar{v}_{x x} \leq 0 \quad \forall(x, t) \in D_{T}$.

Now suppose there exists $\left(x^{*}, t^{*}\right) \in D_{T}$ such that

$\bar{v}\left(x^{*}, t^{*}\right)=0=\sup _{(x, t) \in \bar{D}_{T}} \bar{v}(x, t)$.

Then, via (89), we have that $\bar{v}$ is continuous on $\bar{D}_{T}$ and that $\bar{v}_{x}, \bar{v}_{t}$ and $\bar{v}_{x x}$ exist and are continuous on $D_{T}$. Hence, with (96), we may apply the Strong Parabolic Maximum Principle [16] to $\bar{v}$ on $\bar{D}_{T}$, which requires

$\bar{v}(x, t)=0 \quad \forall(x, t) \in \bar{D}_{t *}$.

Then, using (97) together with (88), (71), (64), (57) and (D2), we have

$u_{t}(x, t)=0 \quad \forall(x, t) \in \bar{D}_{t *}$.

It then follows, via (21) and regularity conditions (R1) and (R2) that

$u(x, t)=1 \quad \forall(x, t) \in \bar{D}_{t *}$. 
However, this contradicts (20). Therefore,

$\bar{v}(x, t)<0 \quad \forall(x, t) \in D_{T}$,

and, via (88), (71), (64), (57) and (D2), we have

$u_{t}(x, t)<0 \quad \forall(x, t) \in D_{T}$.

Since this holds for any $T>0$, we have

$u_{t}(x, t)<0 \quad \forall(x, t) \in D_{\infty}$,

as required.

We next have

Proposition 4 Let $u: \bar{D}_{\infty} \rightarrow \mathbb{R}$ be a solution to $[I B V P]$. Then

$1<D(u(x, t)) u_{x}(x, t)<k \quad \forall(x, t) \in D_{\infty}$.

Proof Fix $t>0$ and let $(x, t) \in D_{\infty}$. Applying the mean value theorem, with $f: \bar{D}_{\infty} \rightarrow \mathbb{R}$ as defined in (55), we have

$f_{x}(x, t)-f_{x}(0, t)=f_{x x}(\theta x, t) x$,

and

$f_{x}(h(t), t)-f_{x}(x, t)=f_{x x}\left(x+\theta^{\prime}(h(t)-x), t\right)(h(t)-x)$,

with $0<\theta, \theta^{\prime}<1$. Now, from (60), we have

$\frac{\varepsilon}{D(u)} f_{t}=f_{x x} \quad$ on $D_{\infty}$.

Thus, via (D2), (57) and Corollary 2, it follows that

$f_{x x}<0$ on $D_{\infty}$.

Consequently, via (98), we have

$f_{x}(x, t)<f_{x}(0, t) \quad \forall(x, t) \in D_{\infty}$,

and, via (58),

$D(u(x, t)) u_{x}(x, t)<D(u(0, t)) u_{x}(0, t) \quad \forall(x, t) \in D_{\infty}$.

Next, using (20) and Proposition 2, we have

$D(u(0, t)) u_{x}(0, t)=k u(0, t) \leq k \quad \forall t \in(0, \infty)$.

Hence, via (101),

$D(u(x, t)) u_{x}(x, t)<k \quad \forall(x, t) \in D_{\infty}$.

Similarly, via (99) and (100), we have

$f_{x}(h(t), t)<f_{x}(x, t) \quad \forall(x, t) \in D_{\infty}$,

which, via (58), becomes

$D(u(x, t)) u_{x}(x, t)>D(u(h(t), t)) u_{x}(h(t), t) \quad \forall(x, t) \in D_{\infty}$. 
Also, using (22) and regularity condition (R1), we have

$D(u(h(t), t)) u_{x}(h(t), t)=h_{t}(t)+1 \geq 1 \quad \forall t \in(0, \infty)$.

Hence, via (103),

$D(u(x, t)) u_{x}(x, t)>1 \quad \forall(x, t) \in D_{\infty}$,

and therefore, with (102), we have

$1<D(u(x, t)) u_{x}(x, t)<k \quad \forall(x, t) \in D_{\infty}$,

as required.

The regularity condition (R2) with (D3) then immediately allows for

Corollary 3 Let $u: \bar{D}_{\infty} \rightarrow \mathbb{R}$ be a solution to $[I B V P]$. Then

$1 \leq D(u(x, t)) u_{x}(x, t) \leq k \quad \forall(x, t) \in \bar{D}_{\infty}$.

In addition, we have

Corollary 4 The existence of a solution to $[I B V P]$ requires $k>1$.

Proof Let $u: \bar{D}_{\infty} \rightarrow \mathbb{R}$ and $h:[0, \infty) \rightarrow \mathbb{R}$ be a solution to [IBVP]. Then from Proposition 4, we have $1<D(u(x, t)) u_{x}(x, t)<k \quad \forall(x, t) \in D_{\infty}$,

which is only possible when $k>1$.

Next we have

Proposition 5 Let $u: \bar{D}_{\infty} \rightarrow \mathbb{R}$ be a solution to [IBVP]. Then

$u(0, t) \geq \frac{1}{k} \quad \forall t \in(0, \infty)$,

$u(x, t)>\frac{1}{k} \forall(x, t) \in \bar{D}_{\infty} \backslash(\{0\} \times(0, \infty))$.

Proof From (20), we have

$k u(0, t)=D(u(0, t)) u_{x}(0, t) \quad \forall t \in(0, \infty)$.

It then follows, from Corollary 3 , that

$u(0, t) \geq \frac{1}{k} \quad \forall t \in(0, \infty)$.

In addition,

$u(x, t)-u(0, t)=\int_{0}^{x} u_{\lambda}(\lambda, t) \mathrm{d} \lambda>0 \quad \forall(x, t) \in \bar{D}_{\infty} \backslash(\{0\} \times[0, \infty))$,

via Corollary 3 and (D2). Thus, from (105) and (104), we have

$u(x, t)>\frac{1}{k} \quad \forall(x, t) \in \bar{D}_{\infty} \backslash(\{0\} \times[0, \infty))$.

However, $u(0,0)=1>k^{-1}$, via Corollary 4 , and so,

$u(x, t)>\frac{1}{k} \quad \forall(x, t) \in \bar{D}_{\infty} \backslash(\{0\} \times(0, \infty))$,

as required. 
We now obtain bounds on $h$ in the following:

Proposition 6 Let $h:[0, \infty) \rightarrow \mathbb{R}$ describe the free boundary in [IBVP]. Then

$0<h(t)<F(1)-F\left(k^{-1}\right) \quad \forall t \in(0, \infty)$.

Proof First, via (24) and (25), we have $h(0)=0$. Also, from (21) and (25) we have $u(0,0)=1$. Hence, via (25), Corollary 4, (20) and (D1) we have

$D(1) u_{x}(0,0)=u_{x}(0,0)=k u(0,0)=k>1$.

Thus, via (22), (25) and (106),

$h_{t}(0)=u_{x}(0,0)-1=k-1>0$.

It then follows from (107), with $h(0)=0$ and regularity condition (R1), that

$h(t)>0 \quad \forall t \in(0, \infty)$.

Next take any $t>0$. It follows from the mean value theorem with (55), (56) and (58) that

$F(u(h(t), t))-F(u(0, t))=F_{x}(u(\hat{\theta} h(t), t)) h(t)=D(u(\hat{\theta} h(t), t)) u_{x}(\hat{\theta} h(t), t) h(t)$,

with $0<\hat{\theta}<1$. Then, via Proposition 4, (108) and (109),

$F(u(h(t), t))-F(u(0, t))>h(t)$,

from which we obtain, via (21),

$h(t)<F(1)-F(u(0, t))$.

However, via (20) and Corollary 3,

$u(0, t)=\frac{D(u(0, t)) u_{x}(0, t)}{k} \geq \frac{1}{k}$.

Therefore, via (110) and (31),

$h(t)<F(1)-F(u(0, t)) \leq F(1)-F\left(k^{-1}\right) \quad \forall t \in(0, \infty)$.

Thus, combining (108) and (111), we have

$0<h(t)<F(1)-F\left(k^{-1}\right) \quad \forall t \in(0, \infty)$,

as required.

Now let $k>1$ and let $u: \bar{D}_{\infty} \rightarrow \mathbb{R}$ and $h:[0, \infty) \rightarrow \mathbb{R}$ be a solution to [IBVP]. It follows from (22), (R1) and Proposition 6 that $h(t)$ is a monotone increasing function of $t$ and is bounded above by $F(1)-F\left(k^{-1}\right)>0$. Consequently, there exists a constant $0<\bar{h} \leq F(1)-F\left(k^{-1}\right)$ such that

$h(t) \rightarrow \bar{h} \quad$ as $t \rightarrow \infty$.

Similarly, Proposition 2, Proposition 3, Corollary 3 and Proposition 5 together with the Ascoli-Arzelà Compactness Theorem establish the existence of a continuous function $\bar{u}:[0, \bar{h}] \rightarrow \mathbb{R}$ such that

$u(x, t) \rightarrow \bar{u}(x) \quad$ as $t \rightarrow \infty$ uniformly for $0 \leq x \leq h(t)$,

with $\bar{u}(x)$ monotone increasing for $x \in[0, \bar{h}]$, and

$\frac{1}{k} \leq \bar{u}(x) \leq 1 \quad \forall x \in[0, \bar{h}]$.

Further, the bounds obtained on $u_{x}, u_{t}$ and $h_{t}$, and consequently bounds on $u_{x x}$ together with the Ascoli-Arzela Theorem allow for a deduction that $\bar{u}_{x}$ and $\bar{u}_{x x}$ exist and are continuous, and moreover, $\bar{u}:[0, \bar{h}] \rightarrow \mathbb{R}$ and $\bar{h}$ must satisfy problem (27)-(30), and so are steady state solutions to [IBVP]. Hence $\bar{u}=u_{\mathrm{s}}$ and $\bar{h}=h_{\mathrm{s}}$, as discussed in Sect. 3.1. It is convenient to summarise the results in this subsection in the following: 
Theorem 1 The existence of a solution to $[I B V P]$ requires $k>1$. With $k>1$, let $u: \bar{D}_{\infty} \rightarrow \mathbb{R}$ and $h:[0, \infty) \rightarrow \mathbb{R}$ be a solution to $[I B V P]$. Then

(i) $\frac{1}{k}<u(x, t)<1 \quad \forall(x, t) \in D_{\infty}$,

(ii) $u_{t}(x, t)<0 \quad \forall(x, t) \in D_{\infty}$,

(iii) $1<D(u(x, t)) u_{x}(x, t)<k \quad \forall(x, t) \in D_{\infty}$,

(iv) $\left(D(u) u_{x}\right)_{x}<0 \quad \forall(x, t) \in D_{\infty}$,

(v) $0<h(t)<F(1)-F\left(k^{-1}\right)$ and $0 \leq h_{t}(t)<k-1 \quad \forall t \in(0, \infty)$,

(vi) $h(t) \rightarrow h_{s}^{-}=F(1)-F\left(k^{-1}\right)$ as $t \rightarrow \infty$,

(vii) $u(x, t) \rightarrow u_{s}^{+}(x)=F^{-1}\left(x+F\left(k^{-1}\right)\right)$ as $t \rightarrow \infty$ uniformly for $0 \leq x \leq h(t)$.

We recall that the limit in (vi) is from below, whilst the limit in (vii) is from above. Also, we note that in physical terms the requirement that $k>1$, for a solution to [IBVP] to exist, requires that the cooling process has to be sufficiently strong in order for the development of a wax layer to initiate. From Theorem 1 (i)-(v), we have obtained a priori bounds for [IBVP] on $u: \bar{D}_{\infty} \rightarrow \mathbb{R}$, the partial derivative $u_{x}: D_{\infty} \rightarrow \mathbb{R}$, together with $h:(0, \infty) \rightarrow \mathbb{R}$. Consequently, both global existence and uniqueness for [IBVP] can be anticipated by adopting an iterative approach to accommodate the quasi-linear terms (see, for example [17]), whilst following, in principle, Cannon and Hill [15]. We next develop the analysis of [IBVP] by considering the structure of the solution as $t \rightarrow 0^{+}$and correspondingly as $t \rightarrow \infty$.

\section{Coordinate expansions}

We begin this section by analysing the structure of the solution to [IBVP] as $t \rightarrow 0^{+}$. After which we consider the structure of the solution to [IBVP] as $t \rightarrow \infty$.

\subsection{Coordinate expansions as $t \rightarrow 0^{+}$}

We consider the structure of the solution to [IBVP] (with $k>1$ ) as $t \rightarrow 0^{+}$. It follows, from (20)-(24), that

$h=O(t), \quad u=1+O(t), \quad x=O(t)$,

as $t \rightarrow 0^{+}$. Therefore, we introduce the scaled coordinate

$X=\frac{x}{t}=O(1) \quad$ as $t \rightarrow 0^{+}$,

and write

$h(t)=t H(t)$,

$u(X, t)=1+t U(X, t)$,

with

$H(t)=H_{1}+H_{2} t+O\left(t^{2}\right)$,

$U(X, t)=U_{1}(X)+U_{2}(X) t+O\left(t^{2}\right)$, 
as $t \rightarrow 0^{+}$with $0 \leq X \leq H(t)$. In terms of $X, t, H$ and $U$, [IBVP] becomes

$$
\begin{array}{ll}
\varepsilon t\left(U-X U_{X}+t U_{t}\right)=\left(D(1+t U) U_{X}\right)_{X} & 0<X<H(t), \quad t>0, \\
D(1+t U) U_{X}=k(1+t U) & X=0, \quad t>0, \\
U=0 & X=H(t), \quad t>0, \\
H+t H_{t}=D(1+t U) U_{X}-1 & X=H(t), \quad t>0, \\
U \text { bounded as } t \rightarrow 0^{+} \text {uniformly for } & 0 \leq X \leq H(t),
\end{array}
$$

$H$ bounded as $t \rightarrow 0^{+}$.

Substituting from (115) and (116) into (117)-(120), we have at leading order

$U_{1}^{\prime \prime}=0 \quad 0<X<H_{1}$,

$U_{1}^{\prime}(0)=k$,

$U_{1}\left(H_{1}\right)=0$,

$H_{1}=U_{1}^{\prime}\left(H_{1}\right)-1$.

The solution to this boundary value problem is readily obtained as

$H_{1}=k-1>0$,

with

$U_{1}(X)=k(X-(k-1)) \quad \forall 0 \leq X \leq k-1$.

Terms at $O(t)$ lead to the following boundary value problem for $H_{2}$ and $U_{2}$

$$
\begin{aligned}
& \left(D^{\prime}(1) U_{1} U_{1}^{\prime}+U_{2}^{\prime}\right)^{\prime}=\varepsilon\left(U_{1}-X U_{1}^{\prime}\right) \quad 0<X<H_{1}, \\
& D^{\prime}(1) U_{1}(0) U_{1}^{\prime}(0)+U_{2}^{\prime}(0)=k U_{1}(0), \\
& U_{1}^{\prime}\left(H_{1}\right) H_{2}+U_{2}\left(H_{1}\right)=0, \\
& \left(2-U_{1}^{\prime \prime}\left(H_{1}\right)\right) H_{2}=D^{\prime}(1) U_{1}\left(H_{1}\right) U_{1}^{\prime}\left(H_{1}\right)+U_{2}^{\prime}\left(H_{1}\right) .
\end{aligned}
$$

After some calculation, we obtain the solution to this boundary value problem as

$$
\begin{aligned}
H_{2}= & -\frac{1}{2} k(k-1)(k+\varepsilon(k-1)), \\
U_{2}(X)=- & \left(\frac{\varepsilon}{2} k(k-1)+\frac{D^{\prime}(1) k^{2}}{2}\right) X^{2}-\left(k^{2}(k-1)-D^{\prime}(1) k^{2}(k-1)\right) X \\
& +k^{2}(k-1)^{2}\left(\frac{\varepsilon-D^{\prime}(1)}{2}+1\right)+\frac{k^{3}}{2}(k-1)+\frac{\varepsilon}{2} k(k-1)^{3} \quad \forall 0 \leq X \leq H_{1} .
\end{aligned}
$$

Thus, the coordinate expansions (113) and (114) as $t \rightarrow 0^{+}$, via (115), (116), (127), (128), (133) and (134), are given by

$$
h(t)=(k-1) t-\frac{k}{2}(k-1)(\varepsilon(k-1)+k) t^{2}+O\left(t^{3}\right) \quad \text { as } t \rightarrow 0^{+},
$$


and

$$
\begin{aligned}
u(X, t)= & 1+(k X-k(k-1)) t+\left(-\left(\frac{\varepsilon}{2} k(k-1)+\frac{D^{\prime}(1) k^{2}}{2}\right) X^{2}\right. \\
& -\left(k^{2}(k-1)-D^{\prime}(1) k^{2}(k-1)\right) X \\
& \left.+k^{2}(k-1)^{2}\left(\frac{\varepsilon-D^{\prime}(1)}{2}+1\right)+\frac{k^{3}}{2}(k-1)+\frac{\varepsilon}{2} k(k-1)^{3}\right) t^{2} \\
& +O\left(t^{3}\right) \text { as } t \rightarrow 0^{+} \text {uniformly for } 0 \leq X \leq t^{-1} h(t) .
\end{aligned}
$$

We obtain, from (135) and (136), that

$h_{t}(t) \rightarrow(k-1) \quad$ as $t \rightarrow 0^{+}$,

whilst recalling (112) we have

$u_{x}(x, t) \rightarrow k$ as $t \rightarrow 0^{+}$uniformly for $0 \leq x \leq h(t)$,

and

$u_{x x}(x, t) \rightarrow-\varepsilon k(k-1)-D^{\prime}(1) k^{2} \quad$ as $t \rightarrow 0^{+}$uniformly for $0 \leq x \leq h(t)$.

\subsection{Coordinate expansions as $t \rightarrow \infty$}

We now consider the structure of the solution to [IBVP] as $t \rightarrow \infty$. From Theorem 1, it follows that $h=h_{\mathrm{s}}+o(1), \quad u=u_{\mathrm{s}}(x)+o(1)$,

as $t \rightarrow \infty$. Thus we write

$h(t)=h_{\mathrm{s}}+\bar{h}(t)$,

$u(x, t)=u_{\mathrm{s}}(x)+\bar{u}(x, t)$,

with

$\bar{h}(t)=o(1) \quad$ as $t \rightarrow \infty$,

$\bar{u}(x, t)=o(1) \quad$ as $t \rightarrow \infty$ uniformly for $0 \leq x \leq h(t)$.

On substituting from (139) and (140) into [IBVP], we obtain the leading order problem for $\bar{u}$ and $\bar{h}$, as

$$
\begin{array}{ll}
\varepsilon \bar{u}_{t}=\left(D\left(u_{\mathrm{s}}(x)\right) \bar{u}\right)_{x x} & 0<x<h_{\mathrm{s}}, \quad t \gg 1, \\
\left(D\left(u_{\mathrm{s}}(x)\right) \bar{u}\right)_{x}=k \bar{u} & x=0, \quad t \gg 1, \\
\bar{u}+\bar{h}=0 & x=h_{\mathrm{s}}, \quad t \gg 1, \\
\bar{h}_{t}=\left(D\left(u_{\mathrm{S}}(x)\right) \bar{u}\right)_{x} & x=h_{\mathrm{s}}, \quad t \gg 1 .
\end{array}
$$

We can eliminate $\bar{h}(t)$ from (141)-(144) to obtain

$$
\begin{array}{cl}
\varepsilon \bar{u}_{t}=\left(D\left(u_{\mathrm{S}}(x)\right) \bar{u}\right)_{x x} & 0<x<h_{\mathrm{s}}, \quad t \gg 1, \\
\left(D\left(u_{\mathrm{S}}(x)\right) \bar{u}\right)_{x}=k \bar{u} & x=0, \quad t \gg 1, \\
\bar{u}_{t}=-\left(D\left(u_{\mathrm{S}}(x)\right) \bar{u}\right)_{x} & x=h_{\mathrm{s}}, \quad t \gg 1,
\end{array}
$$


after which $\bar{h}(t)$ is recovered as

$\bar{h}(t)=-\bar{u}\left(h_{\mathrm{s}}, t\right) ; \quad t \gg 1$.

We look for a solution to (145)-(147) in the form

$\bar{u}(x, t)=\phi(x) \mathrm{e}^{-\lambda t}$

with $\lambda \in \mathbb{C}$ to be determined. After substituting from (149) into (145)-(147), we obtain the linear eigenvalue problem

$$
\begin{array}{ll}
\left(D\left(u_{\mathrm{s}}(x)\right) \phi\right)_{x x}=-\varepsilon \lambda \phi & 0<x<h_{\mathrm{s}}, \\
\left(D\left(u_{\mathrm{s}}(x)\right) \phi\right)_{x}=k \phi & x=0, \\
\lambda \phi=\left(D\left(u_{\mathrm{s}}(x)\right) \phi\right)_{x} & x=h_{\mathrm{s}}
\end{array}
$$

with eigenvalue $\lambda \in \mathbb{C}$. It is now convenient to introduce the function $\psi:\left[0, h_{\mathrm{s}}\right] \rightarrow \mathbb{R}$ defined by

$\psi(x)=D\left(u_{\mathrm{s}}(x)\right) \phi(x) \quad \forall 0 \leq x \leq h_{\mathrm{s}}$,

together with the prescribed function $\Delta:\left[0, h_{\mathrm{s}}\right] \rightarrow \mathbb{R}$ given by

$\Delta(x)=D\left(u_{\mathrm{s}}(x)\right) \quad \forall 0 \leq x \leq h_{\mathrm{s}}$.

Substituting from (153) into (150)-(152) we obtain the equivalent eigenvalue problem,

$\psi^{\prime \prime}+\frac{\varepsilon}{\Delta(x)} \lambda \psi=0 \quad 0<x<h_{\mathrm{s}}$,

$\psi^{\prime}=\frac{k}{D\left(k^{-1}\right)} \psi \quad x=0$,

$\psi^{\prime}=\lambda \psi \quad x=h_{\mathrm{s}}$.

Henceforth, we will refer to the generalised Sturm-Liouville eigenvalue problem given by (155)-(157) as [S-L]. We note that $[\mathrm{S}-\mathrm{L}]$ has the following properties:

(S1) The eigenvalues of [S-L] are all real and may be written as

$$
\begin{gathered}
\lambda_{0}<\lambda_{1}<\lambda_{2}<\cdots<\lambda_{r}<\cdots \\
\text { with } \lambda_{r} \rightarrow \infty \text { as } r \rightarrow \infty .
\end{gathered}
$$

(S2) For $\lambda=\lambda_{r}$ (where $r=0,1,2, \ldots$ ) then the corresponding eigenfunction is real valued, say, $\psi_{r}:\left[0, h_{\mathrm{s}}\right] \rightarrow \mathbb{R}$, and may be normalised so that, $\psi_{r}(0)>0$ and,

$$
\int_{0}^{h_{\mathrm{s}}} \frac{1}{\Delta(\sigma)} \psi_{r}^{2}(\sigma) \mathrm{d} \sigma=1 .
$$

(S3) $\psi_{r}(x)>0$ for all $x \in\left[0, h_{\mathrm{s}}\right]$ if and only if $r=0$.

We are now able to establish the following:

Proposition 7 Let $\lambda_{0} \in \mathbb{R}$ and $\psi_{0}:\left[0, h_{s}\right] \rightarrow \mathbb{R}$ be the zeroth eigenvalue and the corresponding zeroth normalised eigenfunction of $[S-L]$. Then $\lambda_{0}>0$.

Proof In [S-L], we will set $\lambda=\lambda_{0}$ and $\psi(x)=\psi_{0}(x)$, so that

$$
\begin{array}{ll}
\psi_{0}^{\prime \prime}+\frac{\varepsilon}{\Delta(x)} \lambda_{0} \psi_{0}=0 & 0<x<h_{\mathrm{s}}, \\
\psi_{0}^{\prime}=\frac{k}{D\left(k^{-1}\right)} \psi_{0} & x=0, \\
\psi_{0}^{\prime}=\lambda_{0} \psi_{0} & x=h_{\mathrm{s}} .
\end{array}
$$


We first multiply both sides of (159) by $\psi_{0}$ to obtain, after an integration,

$\left[\psi_{0}(x) \psi_{0}^{\prime}(x)\right]_{0}^{h_{\mathrm{s}}}-\int_{0}^{h_{\mathrm{s}}}\left(\psi_{0}^{\prime}(x)\right)^{2} \mathrm{~d} x+\varepsilon \lambda_{0}=0$,

where use has been made of (158). With use of (160) and (161), we obtain from (162),

$\psi_{0}^{2}\left(h_{\mathrm{s}}\right) \lambda_{0}-\frac{k}{D\left(k^{-1}\right)} \psi_{0}^{2}(0)-\int_{0}^{h_{\mathrm{s}}}\left(\psi_{0}^{\prime}(x)\right)^{2} \mathrm{~d} x+\varepsilon \lambda_{0}=0$,

and so, after rearranging,

$\lambda_{0}=\frac{\left(k D\left(k^{-1}\right)^{-1} \psi_{0}^{2}(0)+\int_{0}^{h_{\mathrm{s}}}\left(\psi_{0}^{\prime}(x)\right)^{2} \mathrm{~d} x\right)}{\left(\psi_{0}^{2}\left(h_{\mathrm{s}}\right)+\varepsilon\right)}$.

Hence, via (D2) and (S2) we have $\lambda_{0}>0$, as required.

It is now instructive to consider [S-L] as $\varepsilon \rightarrow 0$. We write the eigenvalues of [S-L] as

$0<\lambda_{0}(\varepsilon)<\lambda_{1}(\varepsilon)<\lambda_{2}(\varepsilon)<\cdots<\lambda_{r}(\varepsilon)<\cdots$

where $\lambda_{r}(\varepsilon) \rightarrow \infty$ as $r \rightarrow \infty$. There are two possibilities as $\varepsilon \rightarrow 0$ :

(i) $\lambda=O(1)$, or (ii) $\lambda=O\left(\varepsilon^{-1}\right)$.

We first consider case (i). Hence we introduce the expansions

$\psi(x, \varepsilon)=\chi_{0}(x)+\varepsilon \chi_{1}(x)+O\left(\varepsilon^{2}\right), \quad x \in\left[0, h_{\mathrm{s}}\right]$,

$\lambda(\varepsilon)=l_{0}+\varepsilon l_{1}+O\left(\varepsilon^{2}\right)$,

as $\varepsilon \rightarrow 0$. After substituting from (163) and (164) into [S-L], we obtain at leading order

$\chi_{0}^{\prime \prime}=0 \quad 0<x<h_{\mathrm{s}}$,

$\chi_{0}^{\prime}=\frac{k \chi_{0}}{D\left(k^{-1}\right)} \quad x=0$,

$\chi_{0}^{\prime}=l_{0} \chi_{0} \quad x=h_{\mathrm{s}}$.

On integrating (165), we obtain

$\chi_{0}(x)=\alpha x+\beta$,

where $\alpha, \beta \in \mathbb{R}$ are constants of integration. Applying condition (166), we have

$\alpha-\frac{k}{D\left(k^{-1}\right)} \beta=0$,

and (168) becomes

$\chi_{0}(x)=\alpha\left(x+\frac{D\left(k^{-1}\right)}{k}\right) \quad \forall 0 \leq x \leq h_{\mathrm{s}}$.

Finally applying condition (167) requires

$\left(l_{0}\left(h_{\mathrm{s}}+\frac{D\left(k^{-1}\right)}{k}\right)-1\right) \alpha=0$,

and so, for a non-trivial solution $(\alpha \neq 0)$, we have

$l_{0}=\frac{k}{\left(D\left(k^{-1}\right)+k h_{\mathrm{s}}\right)}$. 
The constant $\alpha$ is now fixed via the normalisation condition (158) as

$\alpha=\left(\int_{0}^{h_{\mathrm{s}}} \frac{1}{\Delta(s)}\left(s+\frac{D\left(k^{-1}\right)}{k}\right)^{2} \mathrm{~d} s\right)^{-\frac{1}{2}}$.

We observe from (169) and (D2) that $\chi_{0}(x)>0$ for all $x \in\left[0, h_{\mathrm{s}}\right]$. Thus in (163) and (164) it follows from (S3) that $\psi=\psi_{0}$ and $\lambda=\lambda_{0}$, and so we have constructed the lowest eigenvalue and eigenfunction only in case (i). It follows from (S1) that all higher eigenvalues will fall into case (ii), and need not be considered further. In summary, we have, from (164) and (170), that

$\lambda_{0}(\varepsilon)=\frac{k}{\left(D\left(k^{-1}\right)+k h_{\mathrm{s}}\right)}+O(\varepsilon)$,

as $\varepsilon \rightarrow 0$, whilst from (163) and (169), we have

$\psi_{0}(x, \varepsilon)=\alpha\left(x+\frac{D\left(k^{-1}\right)}{k}\right)+O(\varepsilon)$

as $\varepsilon \rightarrow 0$ uniformly for $x \in\left[0, h_{\mathrm{s}}\right]$, with the positive constant $\alpha$ given by (171). We observe that (172) gives

$\lambda_{0}(\varepsilon) \sim \frac{k}{\left(D\left(k^{-1}\right)+(k-1)\left\langle D\left(\left[k^{-1}, 1\right]\right)\right\rangle\right)}$,

as $\varepsilon \rightarrow 0$, where $\left\langle D\left(\left[k^{-1}, 1\right]\right)\right\rangle$ is as defined in (42). Equation (174) highlights the contribution of the variable diffusivity across the steady state layer and in particular the mean of this diffusivity together with the diffusivity closest to the coolant. Finally, returning to (139) and (140) via (148), (149) and (153) (with (S1)-(S3)), we have

$$
\begin{aligned}
u(x, t) & =u_{\mathrm{s}}(x)+u_{\infty} \frac{\psi_{0}(x)}{D\left(u_{\mathrm{s}}(x)\right)} \mathrm{e}^{-\lambda_{0} t}+o\left(\mathrm{e}^{-\lambda_{0} t}\right) \quad \forall 0 \leq x \leq h_{\mathrm{s}}, \\
h(t) & =h_{\mathrm{s}}-u_{\infty} \psi_{0}\left(h_{\mathrm{s}}\right) \mathrm{e}^{-\lambda_{0} t}+o\left(\mathrm{e}^{-\lambda_{0} t}\right)
\end{aligned}
$$

as $t \rightarrow \infty$, with $u_{\infty}$ being a positive (via Theorem 1 (vi) and (vii)) global constant, which remains undetermined in this large- $t$ analysis. The structure of $\lambda_{0}$ and $\psi_{0}(x)$ as $\varepsilon \rightarrow 0$ is given by (172)and (173). The steady state solution is approached through terms exponentially small in $t$, with exponent $\lambda_{0}$, as $t \rightarrow \infty$.

\section{Asymptotic solution to [IBVP] as $\varepsilon \rightarrow 0$}

In many applications, the parameter $\varepsilon$ is small (see, for example [12] and [18]). Therefore, it is of value to consider [IBVP] as a parameter perturbation problem with $0<\varepsilon \ll 1$, and consider its asymptotic solution as $\varepsilon \rightarrow 0$, with $k>1$. We expand the solution to [IBVP] in the form

$$
\begin{aligned}
u(x, t) & =u_{0}(x, t)+\varepsilon u_{1}(x, t)+O\left(\varepsilon^{2}\right), \\
h(t) & =h_{0}(t)+\varepsilon h_{1}(t)+O\left(\varepsilon^{2}\right),
\end{aligned}
$$

as $\varepsilon \rightarrow 0$ with $x, t=O(1)$. On substituting from (177) and (178) into [IBVP], we obtain the following problem at leading order for $u_{0}(x, t)$ and $h_{0}(t)$, namely,

$$
\begin{array}{ll}
\left(F\left(u_{0}\right)\right)_{x x}=0 & 0<x<h_{0}(t), \quad t>0, \\
\left(F\left(u_{0}\right)\right)_{x}=k u_{0} & x=0, \quad t>0, \\
u_{0}=1 & x=h_{0}(t), \quad t>0, \\
1+\left(h_{0}\right)_{t}=\left(F\left(u_{0}\right)\right)_{x} & x=h_{0}(t), \quad t>0, \\
u_{0} \rightarrow 1 \text { as } t \rightarrow 0^{+} \text {uniformly for } & 0 \leq x \leq h_{0}(t), \\
h_{0} \rightarrow 0 \text { as } t \rightarrow 0^{+}, &
\end{array}
$$


where $F: \mathbb{R} \rightarrow \mathbb{R}$ is given by (31). An integration of (179) gives

$F\left(u_{0}(x, t)\right)=A(t) x+B(t) \quad 0 \leq x \leq h_{0}(t), \quad t>0$,

where $A(t)$ and $B(t)$ are smooth functions of $t$ to be determined. Applying condition (180) we require,

$A(t)=k F^{-1}(B(t)) \quad t>0$.

Therefore, after rearranging (186), we can rewrite (185) as

$F\left(u_{0}(x, t)\right)=A(t) x+F\left(k^{-1} A(t)\right) \quad 0 \leq x \leq h_{0}(t), \quad t>0$.

We next apply condition (181) to (187) to obtain

$h_{0}(t)=G(A(t)) \quad t>0$,

where $G: \mathbb{R}^{+} \rightarrow \mathbb{R}$ is such that

$G(\lambda)=\frac{F(1)-F\left(k^{-1} \lambda\right)}{\lambda} \quad \forall \lambda \in \mathbb{R}^{+}$.

It is readily established that the following properties are satisfied:

(G1) $G \in C^{3}\left(\mathbb{R}^{+}\right)$,

(G2) $G(k)=0$,

(G3) $G(\lambda)>0$ for all $\lambda \in(0, k)$,

(G4) $G^{\prime}(\lambda)<0$ for all $\lambda \in(0, k]$.

Finally, we must apply condition (182), which gives

$h_{0 t}(t)=A(t)-1, \quad t>0$.

We observe, from Theorem 1 (v) and (190), that (recalling $k>1$ )

$1 \leq A(t) \leq k \quad \forall t>0$.

Thus, via (G4), (191) and (188), we may deduce that

$0 \leq h_{0}(t) \leq h_{\mathrm{s}}(k) \quad \forall t>0$,

(which is in agreement with Proposition 6, recalling that $h_{\mathrm{s}}(k)=F(1)-F\left(k^{-1}\right)$ ) after which, via (G4), we may invert (188) to obtain

$A(t)=G^{-1}\left(h_{0}(t)\right), \quad t>0$.

Here $G^{-1}:\left[0, h_{\mathrm{s}}(k)\right] \rightarrow[1, k]$ is such that $G^{-1} \in C^{3}\left(\left[0, h_{\mathrm{s}}(k)\right]\right)$, and

$G^{-1}(0)=k, \quad G^{-1}\left(h_{\mathrm{s}}(k)\right)=1$

with

$G_{\lambda}^{-1}(\lambda)<0 \quad \forall \lambda \in\left[0, h_{\mathrm{s}}(k)\right]$.

Therefore, via (190) and (193) (together with the condition (184)), we require that $h_{0}:[0, \infty) \rightarrow \mathbb{R}$ satisfies the autonomous 1-dimensional dynamical system

$h_{0 t}=G^{-1}\left(h_{0}\right)-1, \quad t>0$,

$h_{0}(t) \rightarrow 0^{+}$as $t \rightarrow 0^{+}$.

This problem has a unique solution, say

$h_{0}=H(t), \quad t \geq 0$,

with $H \in C^{3}([0, \infty))$, and 
(H1) $H(0)=0$,

(H2) $H^{\prime}(t)>0$ for all $t \in[0, \infty)$,

(H3) $H(t)=(k-1) t+O\left(t^{2}\right)$ as $t \rightarrow 0^{+}$,

(H4) $H(t) \sim h_{\mathrm{s}}(k)-A_{\infty}(k) \mathrm{e}^{-\mu(k) t}$ as $t \rightarrow \infty$.

Here $A_{\infty}(k)>0$ is a global constant, and

$\mu(k)=\frac{k}{k h_{\mathrm{s}}(k)+F^{\prime}\left(k^{-1}\right)}$.

We observe that (H4) and (198) are in agreement with (176). An implicit form for $H(t)$ is obtained from (196) and (197) as

$\int_{0}^{H(t)} \frac{\mathrm{d} \lambda}{\left(G^{-1}(\lambda)-\lambda\right)}=t, \quad \forall t \geq 0$.

Finally, having determined $h_{0}(t)$, we obtain from (187),

$u_{0}(x, t)=F^{-1}\left(A(t) x+F\left(k^{-1} A(t)\right)\right) ; \quad 0 \leq x \leq h_{0}(t), \quad t \geq 0$,

with $A(t)$ given in terms of $h_{0}(t)$ in (193). It is worth observing from (200) that

$u_{0}(0, t)=k^{-1} A(t)=k^{-1} G^{-1}\left(h_{0}(t)\right), \quad t \geq 0$,

which, via (194) and (195), is monotonic decreasing from unity to $k^{-1}$ with increasing $t$.

\section{Numerical solution to [IBVP]}

In this section, we consider numerical solutions to [IBVP] for comparison with the theory of the previous sections. For [IBVP] with constant diffusivity, the method of fundamental solutions was employed in [1] to provide numerical approximation of the solution to [IBVP]. A useful feature of this method is that a node can be placed at $(x, t)=(0,0)$ in the domain to encapsulate the initial-boundary conditions (23) and (24). However, for non-constant diffusivity, the partial differential equation (19) is quasi-linear, and consequently, the non-linearity precludes numerical methods based on fundamental solutions. Hence, in the current situation, we employ an explicit finite-difference method to provide numerical approximations to $[\mathrm{IBVP}] .{ }^{3}$ We note that although this method is simple to apply, in this setting there are several limitations, primarily due to the representation of conditions (23) and (24). We first transform [IBVP] to a rectangular domain, by introducing

$u(x, t)=v(X, t), \quad X=\frac{x}{h(t)} \forall(x, t) \in D_{\infty} \cup \partial D_{\infty}$.

It then follows from (19)-(22) that [IBVP] becomes

$$
\begin{array}{ll}
\varepsilon h^{2} v_{t}=D(v) v_{X X}+\varepsilon X h_{t} h v_{X}+D^{\prime}(v) v_{X}^{2} & 0<X<1, \quad t>0, \\
D(v) v_{X}=k v h & X=0, \quad t>0, \\
v=1 & X=1, \quad t>0, \\
h h_{t}=v_{X}-h \geq 0 & X=1, \quad t>0,
\end{array}
$$

whilst conditions (23) and (24) are extended, via (135) and (137)-(138) to

$$
\begin{aligned}
h(t) & \sim(k-1) t-\frac{k}{2}(k-1)(\varepsilon(k-1)+k) t^{2} \quad \text { as } t \rightarrow 0^{+} \\
v(X, t) & \sim 1+k h(t)(X-1)-\frac{k}{2} h^{2}(t)\left(\varepsilon(k-1)-D^{\prime}(1) k\right)(X-1)^{2} \quad \text { as } t \rightarrow 0^{+},
\end{aligned}
$$

\footnotetext{
3 The MATLAB files used to perform numerical simulations can be found here https://github.com/JCMUoB/ The-Development-of-a-Wax-Layer-on-the-Interior-Wall-of-a-Circular-Pipe/tree/JCMUoB-resubmitted-manuscript-version.
} 
for $X \in[0,1]$. Due to the degeneracy of (202) at $t=0$, we set the initial conditions for the numerical method at $t=\delta>0$, with $\delta$ sufficiently small so that we can use the asymptotic forms for $h$ and $v$ in (206) and (207) at $t=\delta$, respectively. We refer to the initial-boundary value problem given by (202)-(207) as [IBVP*].

To implement the finite-difference method, we employed a uniform spatial grid with $N_{x}$ grid points to represent the interval $[0,1]$ so that the $i$ th spatial grid points $X_{i}=(i-1) d X$ with $d X=1 /\left(N_{x}-1\right)$. The temporal grid points $t_{j}$, used to represent $[\delta, T]$, were not uniformly spaced, with the time step $d t$ chosen sufficiently small at each step to accommodate numerical stability conditions on the discrete evolution equations for $v_{i, j} \approx v\left(X_{i}, t_{j}\right)$ and $h_{j} \approx h\left(t_{j}\right)$, namely

$\mathrm{d} t \ll \min \left\{\frac{(h \mathrm{~d} X)^{2} \varepsilon}{2 \sup _{X \in[0,1]} D(v(X, t))}, \frac{2(h \mathrm{~d} X)^{2} \varepsilon}{\sup _{X \in[0,1]} D^{\prime}(v(X, t))}, \frac{h \mathrm{~d} X}{(1-k)}\right\}$.

We note that this local stability condition limiting $d t$ relaxes, as $h$ increases. The discretisation of (205), (204), (202) and (203), respectively, in the order that $h_{j+1}$ and $v_{i, j+1}$ are computed, is given by

$$
\begin{aligned}
v_{N_{x}, j}= & 1 \\
h_{j+1}= & h_{j}+\mathrm{d} t\left(\frac{v_{N_{x}, j}-v_{N_{x}-1, j}}{\mathrm{~d} x h_{j}}-1\right) \\
v_{i, j+1}= & v_{i, j}+\frac{\mathrm{d} t}{\varepsilon h_{j}^{2}}\left(D\left(v_{i, j}\right)\left(\frac{v_{i-1, j}-2 v_{i, j}+v_{i+1, j}}{\mathrm{~d} x^{2}}\right)\right. \\
& +\varepsilon X_{i} h_{j}\left(\frac{h_{j+1}-h_{j}}{\mathrm{~d} t}\right)\left(\frac{v_{i+1, j}-v_{i-1, j}}{2 \mathrm{~d} x}\right) \\
& \left.+D^{\prime}\left(v_{i, j}\right)\left(\frac{v_{i+1, j}-v_{i-1, j}}{2 \mathrm{~d} x}\right)^{2}\right) i=2, \ldots, N_{x}-1 ; \\
v_{1, j+1}= & v_{2, j+1}-\frac{k \mathrm{~d} x h_{j+1} v_{2, j+1}}{D\left(v_{2, j+1}\right)} .
\end{aligned}
$$

Using this numerical scheme, we approximated the solution to [IBVP*] in the cases when $D(v)=c+(1-c) v$, with $c=1.0, c=0.7, c=0.4$ and 0.1 . The parameters $k(>1)$ and $\varepsilon(>0)$ were chosen from $k=2,10$ and 20 with $\varepsilon=0.1,0.5$ and 1 . For the discretisation, we found that $N_{x}=161$ was sufficient to achieve good accuracy in all cases. Numerical approximations to [IBVP*] were obtained on domains which extended up to $t=20$, and in all cases, monotone convergence to the steady state solution was observed. Moreover, in all cases, the discrepancy between the numerical approximations for $v$ and $h$ evaluated at the final time level, with their respective steady state solutions, was less than 0.03 and 0.006 , respectively. The 36 simulations took approximately 4 hours to run on a standard laptop with an Intel(R) Core(TM) i5-7Y54 CPU @ 1.20GHz 1.60 GHz processor. See Summary Data ${ }^{4}$ for details related to run-time and 'numerical convergence' times. Also, it should be noted, in Figs. 4a-d and 5a-b that the similarity is due to differential scaling of the numerical axis to allow the complete graph to be recorded. We observe in Fig. 4a-d various qualitative features of solutions to [IBVP]. Specifically, in Fig. 4a-d, we observe that as $\varepsilon$ increases, with $D$ and $k$ fixed, the solutions to [IBVP] converge to the steady state $u_{\mathrm{s}}$ at a slower exponential rate. This is further illustrated in Fig. 5a, b which graph $h$ against $t$ for each of the cases illustrated in Fig. 4a-d. Additionally, in Fig. 4a-d, we observe that as $k$ increases, with $D$ and $\varepsilon$ fixed, the time taken for $u$ to approach the steady state $u_{\mathrm{s}}$ decreases.

We next consider [S-L] given by (155)-(157), and, in particular, we determine the lowest eigenvalue $\lambda_{0}$. For constant $D(=1), \lambda_{0}$ can be determined by an algebraic equation (see Fig. 6a and [1, p. 106] for details). For non-constant $D$, to approximate $\lambda_{0}$ we numerically approximate the boundary condition $(156)$, with $\psi(0)=1$, and

\footnotetext{
${ }^{4}$ Summary Data from numerical simulations is available here https://github.com/JCMUoB/The-Development-of-a-Wax-Layer-on-theInterior-Wall-of-a-Circular-Pipe/tree/JCMUoB-resubmitted-manuscript-version.
} 

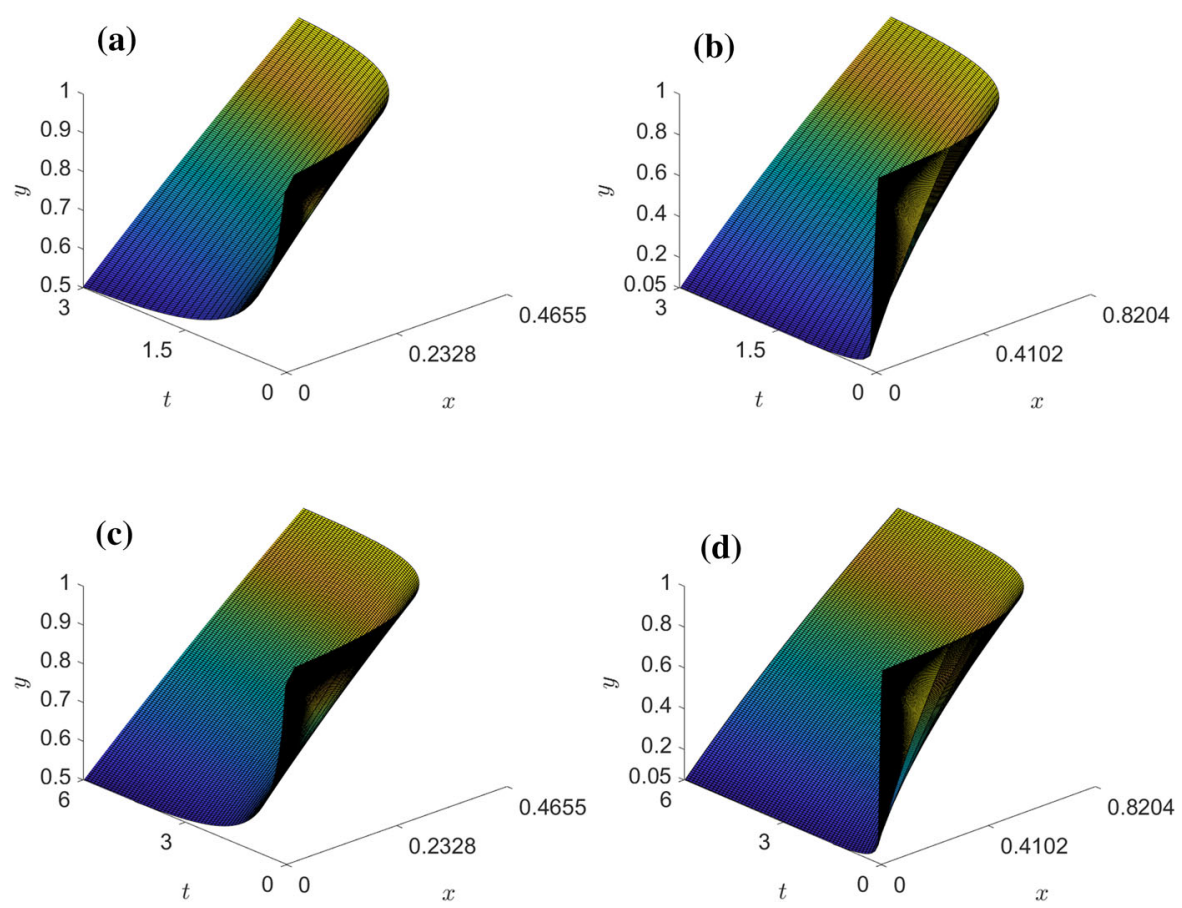

Fig. 4 Plots of $y=u(x, t)$ for $0 \leq x \leq h(t)$ with $u$ and $h$ being the numerical solution to [IBVP], obtained from $v_{i, j}$ and $h_{j}$. In a-d, we illustrate surface plots for $D(u)=0.7+0.3 u$ with $\mathbf{a} k=2$ and $\varepsilon=0.1$; $b=20$ and $\varepsilon=0.1$; $k=2$ and $\varepsilon=1$; and $\mathbf{d} k=20$ and $\varepsilon=1$
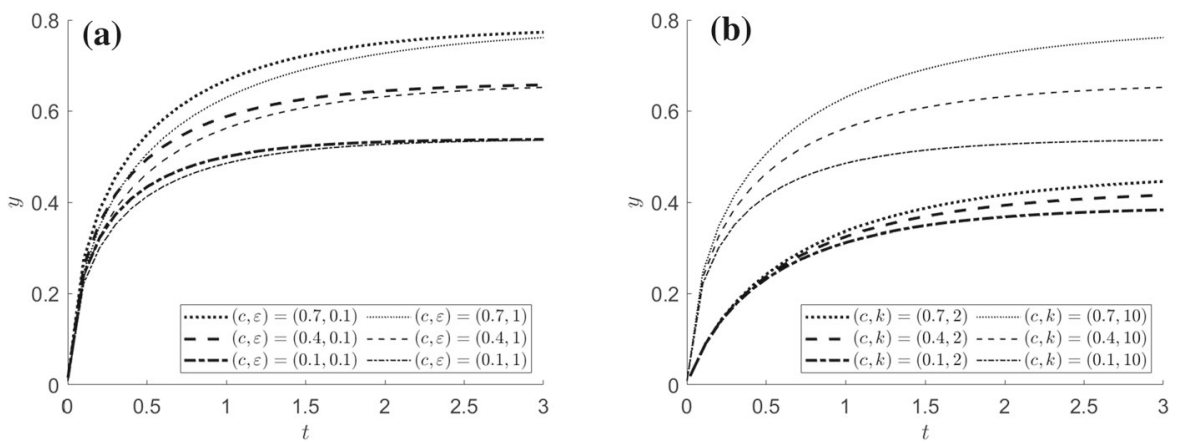

Fig. 5 Plots of the free boundary component of the numerical solution to [IBVP*], $y=h(t)$. Time domains are chosen to highlight the discrepancy in time taken for $h$ to get close to its steady state $h_{\mathrm{s}}$. Here we consider [IBVP*] with $D(u)=c+(1-c) u$ and a $k=10$ with $(c, \varepsilon)$ varying, and $\mathbf{b} \varepsilon=1$ with $(c, k)$ varying

then employ a shooting method which numerically approximates (155) with an explicit Euler method. We combine this with a bisection method using criteria (157) to numerically approximate $\lambda_{0}$ for $D(u)=0.7+0.3 u$ (see Fig. 6b). Specifically, 1056 eigenvalues are represented in Fig. 6 which are computed to 0.001 precision using $N_{x}=161$ grid nodes as above, to represent $\psi$ in each case. On the computer referred to above, it took approximately 6 hours to complete the eigenvalue computations to produce Fig. $6 \mathrm{~b}$.

The eigenvalue surfaces for [S-L] in Fig. 6a, b are qualitatively different. The surface for $D=1$ is concave up, in contrast to the surface for $D(u)=0.7+0.3 u$ which is not concave. As $\varepsilon \rightarrow 0^{+}$the asymptotic form of (172) is well represented by the numerical solution. We conclude from this that the rate of convergence of the evolving crystallised wax layer to its steady state is significantly affected by the variation in crystallised wax conductivity. 

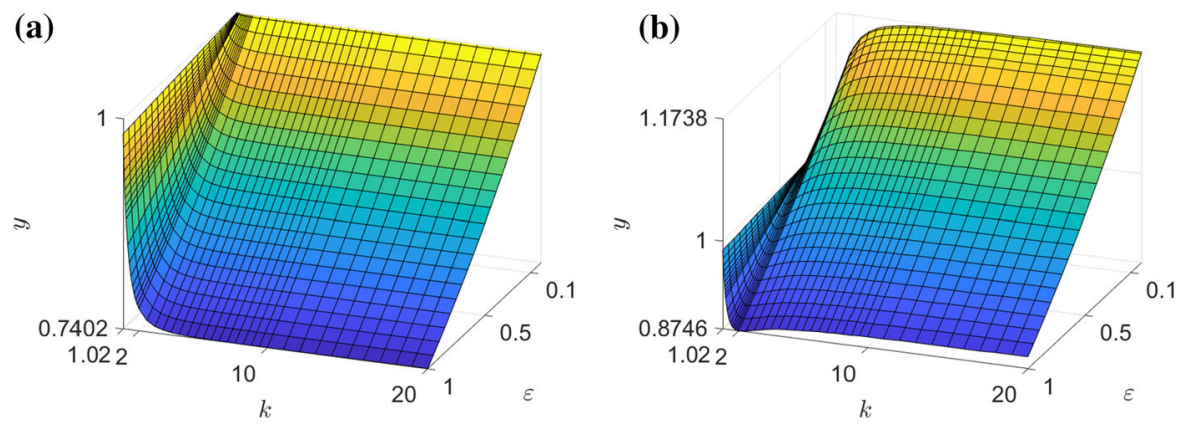

Fig. 6 Plots of the surface $y=\lambda_{0}(\varepsilon, k)$ for $D(u)=c+(1-c) u$ with $\mathbf{a} c=1$ and $\mathbf{b} c=0.7$

\section{Comparison with experiments}

Detailed experiments on the formation of wax-deposited layers in straight circular pipes transporting heated oil, when subject to external wall cooling, have been performed and reported by Hoffman and Amundsen [5]. In this section, we make qualitative and trend comparisons with the theory presented in this paper and the experimental results in [5]. We observe that the experiments reported in [5] have fixed paraffinic wax and oil properties in all experiments, whilst the bulk oil temperature $T_{\mathrm{o}}$, the coolant temperature, $T_{\mathrm{c}}$ and the oil flow Nusselt number $N_{u}$ are varied in turn, with a series of experiments performed in each case and wax layer evolving profile and equilibrium thickness measured. First, we relate the variations in $T_{\mathrm{o}}, T_{\mathrm{c}}$ and $N_{u}$, respectively, to the dimensionless parameters in the model, namely $\varepsilon$ and $k$. We observe from (17) that, first $k>1$ for wax layer formation to occur, and thereafter,

(a) Increasing $T_{\mathrm{o}}$ decreases $k$ whilst keeping $\varepsilon$ fixed.

(b) Decreasing $T_{\mathrm{c}}$ increases $k$ and $\varepsilon$ together.

(c) Increasing $N_{u}$ decreases $k$ whilst leaving $\varepsilon$ fixed.

Thus, via Sect. 6, we can refer, for comparison, to the evolution of the dimensionless wax layer thickness with dimensionless time in Figs. 5a and b. Figure 5b corresponds to cases (a) and (c) above. Additionally, Fig. 5a and $\mathrm{b}$ when combined correspond to case (b) above. The purpose of this section is now to verify that the outcomes of the theory developed in this paper, in terms of the spatiotemporal dynamics of the evolving wax layer, and its long time steady state form (as detailed in Sects. 2-6) do, in fact, capture the principle features of the dynamics of wax layer formation, as recorded in a number of experimental and observational studies. For definiteness in this comparison, we make comparisons between the numerically determined model wax layer properties of Sect. 6, and the experimentally reported results in [5].

We first consider Fig. 11 in [5]. This graphs the experimental wax layer equilibrium height against the wall temperature $T_{\mathrm{c}}$, at two different values of $N_{u}$. Each graph has a critical value of $T_{\mathrm{c}}$, above which a wax layer does not form. This is consistent with the theory corresponding to the critical value $k=1$. As $T_{\mathrm{c}}$ decreases from the critical value, the equilibrium wax layer thickness increases; this is born out for the theory in Fig. 5b.

We now consider Fig. 15 in [5], which graphs the evolution of the wax layer thickness with time, for a number of increasing oil temperatures $T_{\mathrm{0}}$. These profiles show remarkably similar structure to those theoretical profiles in Fig. 5a and $\mathrm{b}$. The experimental graphs show a lowering of the profile with increasing $T_{\mathrm{o}}$. These can be compared with Fig. 5b, which show lowering profiles with decreasing $k$. This is consistent via (a). Note that the experiments show that a wax layer will not form for $T_{\mathrm{O}}$ sufficiently large. This corresponds to $k$ decreasing to $k=1$ in the theory.

Finally, we consider Fig. 18 in [5]. This shows the evolution of the wax layer thickness with time, for a number of Nusselt numbers, with $N_{u}$ increasing. We see that the wax layer profiles are lowering with increasing Nusselt number. This corresponds to case (c) in the theory, and we compare with Fig. 5b. We see that, with $\varepsilon$ fixed, the corresponding profiles are lowering as $k$ decreases, in line with the experiments. To conclude, we note the striking similarity between the experimental profiles in Fig. 18 of [5] and the model profiles given in Fig. 5. 


\section{Conclusions}

In this paper, we have developed and analysed in detail the simple thermal model for the development of a wax layer on the interior wall of a circular pipe transporting heated oil containing dissolved paraffinic wax, which was introduced in [1]. This approach is gaining considerable traction compared to the traditional mechanical and material diffusion theories; it is able to describe features associated with wax layer formation which have been absent from, or even contrary to, the outcomes from the mechanical and material diffusion theories. This view point is vindicated in a number of recent detailed reviews of the wax layer formation process, see, for example $[4,9,12]$ and $[10]$. The current paper extends the theory developed in [1] to allow for the dependence of solid wax thermal conductivity on local temperature, which is a significant feature of solidified paraffinic wax, and which should be included as a principle component in the thermal model. The initial comparisons with experimental results in [5] considered in this paper are very encouraging for the thermal mechanism model introduced in [1] and developed here. A key feature identified, which is principally associated with the crystallised wax temperature-dependent conductivity, is the effect this has on the time scale to reach steady state. A more detailed experiment with model comparison to consider quantitative agreement appears to be a worthwhile and appropriate endeavour to undertake. In addition, we make the final observation that pipelines in the field are exceptionally long in general, and under such field conditions, it is to be expected that core temperature reduction with axial distance will play a significant role in the axial variation of wax layer formation. The authors are considering this development at present.

Acknowledgements The authors would like to thank Ruben Schulkes (Statoil Petroleum, AS) for his helpful comments. We also thank the referees for very useful comments.

\section{Declarations}

Conflict of interest The authors declare that they have no conflict of interest.

Open Access This article is licensed under a Creative Commons Attribution 4.0 International License, which permits use, sharing, adaptation, distribution and reproduction in any medium or format, as long as you give appropriate credit to the original author(s) and the source, provide a link to the Creative Commons licence, and indicate if changes were made. The images or other third party material in this article are included in the article's Creative Commons licence, unless indicated otherwise in a credit line to the material. If material is not included in the article's Creative Commons licence and your intended use is not permitted by statutory regulation or exceeds the permitted use, you will need to obtain permission directly from the copyright holder. To view a copy of this licence, visit http://creativecommons.org/licenses/by/4.0/.

\section{References}

1. Needham DJ, Johansson BT, Reeve T (2014) The development of a wax layer on the interior wall of a circular pipe transporting heated oil. QJMAM 67:93-125

2. Schulkes RMSM (2011) Modelling wax deposition as a Stefan problem. Unpublished notes

3. Azevedo LFA, Teixeira AM (2003) A critical review of the modeling of wax deposition mechanisms. Pet Sci Technol 21:393-408

4. Mahir LHA (2020) Modelling paraffin wax deposition from flowing oil onto cold surfaces. Ph.D. Thesis, University of Michigan

5. Hoffman R, Amundsen L (2010) Single-phase wax deposition experiments. Energy Fuels 24:1069-1080

6. Halstensen M, Arvoh BK, Amundsen L, Hoffman R (2013) Online estimation of wax deposition thickness in single phase sub-sea pipelines based on acoustic chemometrics: a feasibility study. Fuel 105:718-727

7. Singh P, Venkatesan R, Fogler HS, Nagaragan NR (2000) Formation and aging of incipient thin film wax-oil gels. AICLE J 46(5):1059-1074

8. Singh P, Venkatesan R, Fogler HS, Nagaragan NR (2000) Aging and morphological evolution of wax-oil gels during externally cooled flow through pipes. In: Second international conference in petroleum phase behaviour and fouling. Copenhagen, Denmark

9. Mehrotra AK, Ehsani S, Haj-Shaflei S, Kasuma S (2020) A review of heat-transfer mechanism for solid deposition from 'waxy' or paraffinic mixtures. Can J Chem Eng 98(12):2463-2488

10. van der Geest C, Melchuna A, Bizarre L, Bannwart AC, Guersoni VCB (2021) Critical review on wax deposition in single-phase flow. Fuel 293:120358 
11. Viega HMB, Fleming FP, Azevedo LFA (2017) Wax deposit thermal conductivity measurement under flowing conditions. Energy Fuels 31:11532-11547

12. Kaye GWC, Laby TH, Mechanical properties of materials. Tables of physics chemical constants. National Physical Laboratory (NPL) (archived from the original on 11/03/2008)

13. Friedman A (1982) Variational principles and free-boundary problems. Wiley, New York

14. Schatz A (1969) Free boundary problems of Stephan type with prescribed flux. J Math Anal Appl 28:569-580

15. Cannon JR, Hill CD (1967) Existence, uniqueness, stability, and monotone dependence in a Stefan problem for the heat equation. J Math Mech 17:1-20

16. Walter W (1986) On the strong maximum principle for parabolic differential equations. Proc Edinb Math Soc 29:93-96

17. Ladyženskaja OA, Solonnikov VA, Ural'ceva NN, (1968) Linear and quasi-linear equations of parabolic type. AMS, Rhode Island

18. Burger ED, Perkins TK, Striegler JH (1981) Studies of wax deposition in the trans Alaska pipeline. J Petrol Technol 33:1075-1086

Publisher's Note Springer Nature remains neutral with regard to jurisdictional claims in published maps and institutional affiliations. 